\title{
HEAVY-ELEMENT DISPERSION IN THE METAL-POOR GLOBULAR CLUSTER M92*
}

\author{
IAN U. ROEDERER ${ }^{1,3}$ AND CHRISTOPHER SNEDEN ${ }^{2}$ \\ ${ }^{1}$ Carnegie Observatories, 813 Santa Barbara Street, Pasadena, CA 91101, USA; iur@obs.carnegiescience.edu \\ ${ }^{2}$ Department of Astronomy, University of Texas at Austin, 1 University Station, C1400, Austin, TX 78712, USA \\ Received 2011 February 28; accepted 2011 April 25; published 2011 June 9
}

\begin{abstract}
Dispersion among the light elements is common in globular clusters (GCs), while dispersion among heavier elements is less common. We present detection of $r$-process dispersion relative to $\mathrm{Fe}$ in 19 red giants of the metal-poor GC M92. Using spectra obtained with the Hydra multi-object spectrograph on the WIYN Telescope at Kitt Peak National Observatory, we derive differential abundances for 21 species of 19 elements. The Fe-group elements, plus $\mathrm{Y}$ and $\mathrm{Zr}$, are homogeneous at a level of 0.07-0.16 dex. The heavy-elements La, Eu, and Ho exhibit clear star-to-star dispersion spanning 0.5-0.8 dex. The abundances of these elements are correlated with one another, and we demonstrate that they were produced by $r$-process nucleosynthesis. This $r$-process dispersion is not correlated with the dispersion in $\mathrm{C}, \mathrm{N}$, or $\mathrm{Na}$ in $\mathrm{M} 92$, indicating that $r$-process inhomogeneities were present in the gas throughout star formation. The $r$-process dispersion is similar to that previously observed in the metal-poor GC M15, but its origin in M15 or M92 is unknown at present.
\end{abstract}

Key words: globular clusters: individual (NGC 6341) - nuclear reactions, nucleosynthesis, abundances - stars: abundances - stars: Population II

Online-only material: color figures, machine-readable and VO tables

\section{INTRODUCTION}

Dispersion observed among the light elements ( $\mathrm{Li}, \mathrm{C}, \mathrm{N}, \mathrm{O}$, $\mathrm{Na}, \mathrm{Mg}, \mathrm{Al}$, and $\mathrm{Si}$ ) in Galactic globular clusters (GCs) has motivated numerous attempts to characterize it, both in terms of the internal star-to-star dispersion and the range from one $\mathrm{GC}$ to another. An order of magnitude increase in the amount of observational data of these elements in the last five years has led to an explosion of attempts to model the light-element dispersion and understand its implications for GC formation. The uniformity of heavier $\alpha$, Fe-group, and neutron $(n)$ capture elements in GCs has provided important constraints for these models, but characterizing this homogeneity has usually been of secondary importance when designing observational studies.

Instruments for multi-object observations ( $\sim 20-100$ stars per GC) dictate that a choice of wavelength range must be made. Wavelength ranges appropriate for the $\mathrm{O}-\mathrm{Al}$ absorption lines have allowed simultaneous study of heavier elements only when their absorption lines fortuitously fall in the same wavelength range. Other studies have examined many elements per star by obtaining complete wavelength coverage and high spectral resolution at the cost of studying a limited number of stars ( 5-20 stars per GC). Together, these approaches have allowed observers to identify infrequent but genuine dispersion among the heavy elements.

For example, several massive GCs exhibit significant subpopulations of stars whose $\mathrm{Ca}$ - or Fe-group abundances are different from one another (M22, e.g., Marino et al. 2011 and references therein; M54, e.g., Carretta et al. 2010a and references therein; NGC 1851, e.g., Carretta et al. 2010b and

\footnotetext{
* The WIYN Observatory is a joint facility of the University of Wisconsin-Madison, Indiana University, Yale University, and the National Optical Astronomy Observatory.

3 Visiting Astronomer, Kitt Peak National Observatory, National Optical Astronomy Observatory, which is operated by the Association of Universities for Research in Astronomy, Inc. (AURA), under cooperative agreement with the National Science Foundation.
}

references therein; NGC 2419, Cohen et al. 2010; $\omega$ Centauri, e.g., Johnson \& Pilachowski 2010 and references therein). Two studies have reported individual stars in M92 whose Fegroup abundances are higher by $0.15-0.20$ dex than other members (King et al. 1998; Langer et al. 1998). While the heaviest elements in most metal-poor GCs have been produced primarily by rapid $(r) n$-capture nucleosynthesis, some GCs have been enriched by a significant amount of material produced in the slow $(s) n$-capture process (M4, Ivans et al. 1999, Yong et al. 2008a, 2008b, 2009; M22, Marino et al. 2009, 2011; NGC 1851, Yong \& Grundahl 2008, Carretta et al. 2010b, Villanova et al. 2010; $\omega$ Centauri, e.g., Smith et al. 2000, Johnson \& Pilachowski 2010). Finally, the $n$-capture elements in M15, produced by $r$-process nucleosynthesis, exhibit significant starto-star dispersion (nearly $\sim 1$ dex; Sneden et al. 1997, 2000; Otsuki et al. 2006; Sobeck et al. 2011).

How anomalous is M15? In this paper we revisit the heavy $n$-capture element abundances in M92, a metal-poor GC similar (in metallicity, age, luminosity, and orbital kinematics) to M15. Table 1 summarizes the basic properties of M92. The $n$-capture elements in M92 are relatively understudied considering that it is one of the brightest and most metal-poor GCs accessible to northern hemisphere telescopes.

Cohen (1979) performed the first study of $n$-capture elements in M92, deriving abundances of Y II, Zr II, Ba II, La II, and Nd II in four red giant branch (RGB) stars. She found a general decrease in these abundances relative to the more metal-rich GC M13, but the overall pattern was unchanged. Peterson et al. (1990) derived abundances of $Y_{\text {II }}$ and Ba II in 2 M92 RGB stars. Armosky et al. (1994) examined Ba II and Nd II in nine and four RGB stars, respectively. That study found no dispersion in either element and, accordingly, no correlation with the light-element dispersion in M92. At this point, the observations were still inadequate to discern the nucleosynthetic origin of the heavy elements in M92.

Shetrone (1996) and Shetrone et al. (1998) derived abundances of Eu II in three RGB stars and Ba II in five RGB stars, 
Table 1

M92 Basic Parameters

\begin{tabular}{lll}
\hline \hline Quantity & \multicolumn{1}{c}{ Value } & References \\
\hline R.A. (J2000) & $17: 17: 07$ & 1 \\
Decl. (J2000) & $+43: 08: 11$ & 1 \\
$\ell$ & 68.3 & 1 \\
$b$ & 34.9 & 1 \\
$M_{V}$ & -8.20 & $1,2,3,4$ \\
$(m-M)_{V}$ & $14.67 \pm 0.08$ & 5 \\
$E(B-V)$ & 0.02 & $1,6,7,8,9$ \\
$R_{\odot}$ & $8.2 \mathrm{kpc}$ & 1 \\
$R_{\mathrm{G} . \mathrm{C} .}$ & $9.6 \mathrm{kpc}$ & 1 \\
$R_{\text {peri }}$ & $1.4 \pm 0.2 \mathrm{kpc}$ & 10 \\
$R_{\text {apo }}$ & $9.9 \pm 0.4 \mathrm{kpc}$ & 10 \\
$Z_{\text {max }}$ & $3.8 \pm 0.5 \mathrm{kpc}$ & 10 \\
$T_{\text {orbit }}$ & $0.20 \pm 0.01 \mathrm{Gyr}$ & 10 \\
\hline
\end{tabular}

References. (1) Harris 1996; (2) Webbink 1985; (3) Peterson \& Reed 1987; (4) van den Bergh et al. 1991; (5) Pont et al. 1998; (6) Sandage 1969; (7) Zinn 1980; (8) Reed et al. 1988; (9) Schlegel et al. 1998; (10) Dinescu et al. 1999.

respectively. Sneden et al. (1997) used the Ba from Armosky et al. (1994) and the $\mathrm{Eu}$ from Shetrone (1996) to infer that $r$-process nucleosynthesis dominated the production of the heavy elements in M92 (and M13) more than in the solar system (S.S.). Sneden et al. (2000) derived Ba II abundances for 32 stars in M92; the dispersion in [Ba/Fe], ${ }^{4} 0.16 \mathrm{dex}$, was only slightly less than that of 31 stars in M15, 0.21 dex.

Over the last 10 years, a few other investigators (Shetrone et al. 2001; Johnson 2002; Sadakane et al. 2004) have made detailed abundance analyses of small numbers of M92 giants, but no study has examined enough stars to show conclusively whether a dispersion exists among the heaviest elements. This is our motivation for the present study. Sections 2 and 3 describe the characteristics of the new M92 spectra obtained for this study and the details of our abundance analysis. Section 4 presents evidence that genuine dispersion exists among the $n$-capture elements. Section 5 demonstrates that the heavy elements in M92 were produced by $r$-process nucleosynthesis and compares the M92 dispersion with that in M15. We present our conclusions in Section 6.

\section{OBSERVATIONS}

Previous studies of GCs have generally focused on red regions of the spectrum (5600-7000 $)$ ) that are nearer to the peak of the stellar flux distribution and allow study of the light-element dispersion. Since many useful transitions of $n$-capture elements are in the blue around $4000 \AA$, we study this spectral region instead. All observations were taken 2010 May 28-31 using the Hydra multi-object fiber positioner and bench spectrograph (Barden \& Armandroff 1995; Bershady et al. 2008) on the WIYN 3.5 m Telescope at Kitt Peak National Observatory. We used the blue fiber cable (3".1 fibers on sky) and 316@63.4 echelle grating to obtain a resolution of $R \equiv \lambda / \Delta \lambda \sim 14,000$ as measured from isolated ThAr lines. The X7.5 filter provides wavelength coverage from $3850<\lambda<4050 \AA$ with steep dropoff beyond these limits.

Rees (1992) measured proper motions for 365 stars with $V \lesssim 16$ in the M92 field, and this is our primary source

\footnotetext{
4 For elements $\mathrm{X}$ and $\mathrm{Y},[\mathrm{X} / \mathrm{Y}] \equiv \log _{10}\left(N_{\mathrm{X}} / N_{\mathrm{Y}}\right)_{\star}-\log _{10}\left(N_{\mathrm{X}} / N_{\mathrm{Y}}\right)_{\odot}$ and
} $\log \epsilon(\mathrm{X}) \equiv \log _{10}\left(\mathrm{~N}_{\mathrm{X}} / \mathrm{N}_{\mathrm{H}}\right)+12.0$.

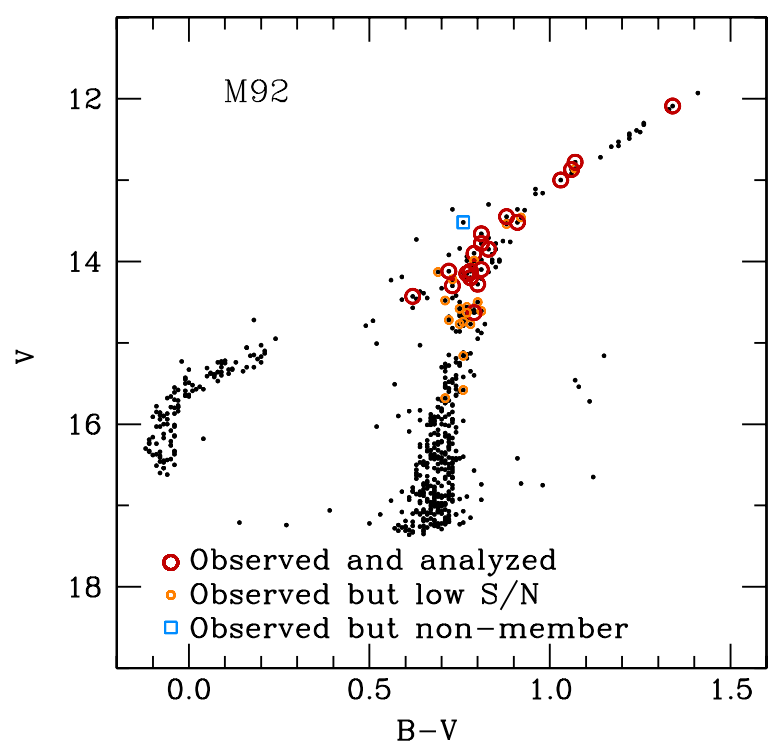

Figure 1. CMD of the RGB, HB, and AGB in M92 with photometry from Buonanno et al. (1983). Stars with large red circles indicate stars observed by us with sufficient $\mathrm{S} / \mathrm{N}$ to perform an abundance analysis, stars with small orange circles indicate stars observed by us that lack the $\mathrm{S} / \mathrm{N}$ necessary to perform an abundance analysis, and the blue square indicates the non-member star VI-7.

(A color version of this figure is available in the online journal.)

for assessing GC membership probability. We used two fiber position settings to observe a total of 39 stars classified as proper motion members $(P>90 \%)$. These stars are marked on the color-magnitude diagram (CMD) shown in Figure 1. Remaining unused fibers were placed on the blank sky to assist in sky subtraction. Most stars (28) were included in both fiber settings, but to increase the total number of stars observed a few additional stars were substituted for the brightest ones after they had achieved adequate signal-to-noise ratios (S/Ns). Exposure times are listed in Table $2 . \mathrm{S} / \mathrm{Ns}$ per pixel in the continuum near $4000 \AA$, derived assuming Poisson statistics, are also listed in Table 2. The $\mathrm{S} / \mathrm{N}$ ranges from $110 / 1$ for the brightest star $(V=12.1)$ to $30 / 1$ for the faintest star $(V=15.7)$. A few stars, such as $\mathrm{V}-45$, have lower $\mathrm{S} / \mathrm{N}$ than would be expected based on their magnitudes; our fiber position astrometry may have been slightly in error for these stars.

Buonanno et al. (1983) is our primary source for broadband $B V$ photometry. They provide a nearly complete census of stars along the RGB, horizontal branch (HB), and asymptotic giant branch (AGB) in M92 in a $14 \times 14$ arcmin field centered on M92 (excluding the crowded central region). A few stars not covered by Buonanno et al. were also observed. Rees (1992) provided $B V$ photometry for these six stars, and the conversion from the Rees to Buonanno et al. scale is $\Delta V \approx 0.00 \pm 0.05$ for the stars in this magnitude range (see Figure 1 of Rees 1992), so we apply no correction. In Section 3.2 we assess the impact of mixing photometric scales on the derived model atmosphere parameters. Finally, $J H K$ photometry from the Two Micron All Sky Survey (2MASS; Skrutskie et al. 2006) is available for nearly all (34) stars in our study. Photometry for our targets is listed in Table 2, which also gives the cross-identification between Sandage \& Walker (1966) and Buonanno et al. (1983).

We use the IRAF environment ${ }^{5}$ to perform standard data reduction, including bias subtraction, flat fielding, image

\footnotetext{
5 IRAF is distributed by the National Optical Astronomy Observatory, which is operated by the Association of Universities for Research in Astronomy, Inc., under cooperative agreement with the National Science Foundation.
} 
Table 2

Photometry, Exposure Times, and S/N Estimates

\begin{tabular}{|c|c|c|c|c|c|c|c|c|c|c|}
\hline $\begin{array}{l}\text { Star Name } \\
\text { (Ref. 1) }\end{array}$ & $\begin{array}{l}\text { Alt. Name } \\
\text { (Ref. 2) }\end{array}$ & $V$ & $B-V$ & $V-J$ & $V-H$ & $V-K$ & Refs. & $\begin{array}{l}\text { No. } \\
\text { Exp. }\end{array}$ & $\begin{array}{c}\text { Exp. Time } \\
\text { (s) }\end{array}$ & $\begin{array}{c}\mathrm{S} / \mathrm{N} \\
(4000 \AA)\end{array}$ \\
\hline VII-18 & Bu488 & 12.09 & 1.34 & 2.20 & 2.86 & 2.97 & 2,4 & 4 & 52700 & $110 / 1$ \\
\hline XII-8 & Bu510 & 12.78 & 1.07 & 2.05 & 2.65 & 2.74 & 2,4 & 4 & 52700 & $100 / 1$ \\
\hline$V-45$ & Bu92 & 12.86 & 1.07 & 2.08 & 2.64 & 2.76 & 2,4 & 4 & 52700 & $35 / 1$ \\
\hline XI-19 & Bu550 & 12.87 & 1.06 & 2.01 & 2.56 & 2.69 & 2,4 & 4 & 52700 & $95 / 1$ \\
\hline XI-80 & $\mathrm{Bu} 454$ & 13.00 & 1.03 & 2.03 & 2.62 & 2.71 & 2,4 & 4 & 52700 & $90 / 1$ \\
\hline XII-34 & Bu330 & 13.45 & 0.88 & 1.85 & 2.38 & 2.46 & 2,4 & 4 & 52700 & $80 / 1$ \\
\hline IV-10 & Bu21 & 13.46 & 0.92 & 1.93 & 2.49 & 2.60 & 2,4 & 4 & 52700 & $20 / 1$ \\
\hline IV-79 & Bu109 & 13.52 & 0.91 & 1.95 & 2.51 & 2.60 & 2,4 & 4 & 52700 & $50 / 1$ \\
\hline VI-7 & $\ldots$ & 13.52 & 0.76 & 1.40 & 1.85 & 1.94 & 3,4 & 8 & 106300 & $105 / 1$ \\
\hline IV-2 & Bu12 & 13.54 & 0.88 & 1.85 & 2.43 & 2.52 & 2,4 & 8 & 106300 & $55 / 1$ \\
\hline VII-10 & $\ldots$ & 13.66 & 0.81 & $\ldots$ & $\ldots$ & $\ldots$ & 3 & 8 & 106300 & $80 / 1$ \\
\hline VI-18 & $\mathrm{Bu} 271$ & 13.78 & 0.81 & 1.80 & 2.34 & 2.38 & 2,4 & 8 & 106300 & $85 / 1$ \\
\hline IX-49 & Bu539 & 13.85 & 0.83 & 1.80 & 2.30 & 2.43 & 2,4 & 8 & 106300 & $85 / 1$ \\
\hline IV-40 & Bu43 & 13.90 & 0.79 & 1.78 & 2.29 & 2.34 & 2,4 & 8 & 106300 & $70 / 1$ \\
\hline XII-31 & Bu322 & 13.99 & 0.79 & 1.81 & 2.31 & 2.43 & 2,4 & 4 & 53600 & $45 / 1$ \\
\hline VIII-44 & Bu545 & 14.10 & 0.81 & 1.76 & 2.30 & 2.41 & 2,4 & 8 & 106300 & $80 / 1$ \\
\hline XI-10 & Bu395 & 14.12 & 0.78 & 1.74 & 2.29 & 2.35 & 2,4 & 8 & 106300 & $75 / 1$ \\
\hline VIII-24 & Bu507 & 14.12 & 0.72 & 1.67 & 2.15 & 2.21 & 2,4 & 8 & 106300 & $70 / 1$ \\
\hline III-4 & Bu14 & 14.13 & 0.69 & 1.59 & 2.06 & 2.14 & 2,4 & 8 & 106300 & $50 / 1$ \\
\hline$\ldots$ & Bu166 & 14.15 & 0.77 & 1.80 & 2.32 & 2.39 & 2,4 & 8 & 106300 & $65 / 1$ \\
\hline IX-89 & Bu497 & 14.20 & 0.78 & 1.74 & 2.27 & 2.37 & 2,4 & 8 & 106300 & $65 / 1$ \\
\hline VII-79 & Bu429 & 14.22 & 0.73 & 1.78 & 2.27 & 2.38 & 2,4 & 8 & 106300 & $65 / 1$ \\
\hline II-39 & Bu71 & 14.28 & 0.80 & 1.66 & 2.16 & 2.28 & 2,4 & 8 & 106300 & $60 / 1$ \\
\hline VII-68 & Bu305 & 14.30 & 0.73 & 1.79 & 2.37 & 2.38 & 2,4 & 8 & 106300 & $70 / 1$ \\
\hline XII-18 & Bu386 & 14.43 & 0.62 & 1.49 & 1.94 & 2.04 & 2,4 & 8 & 106300 & $70 / 1$ \\
\hline V-78 & Bu104 & 14.48 & 0.71 & 1.54 & 1.98 & 2.07 & 2,4 & 8 & 106300 & $55 / 1$ \\
\hline II-24 & Bu37 & 14.50 & 0.80 & 1.63 & 2.16 & 2.23 & 2,4 & 8 & 106300 & $30 / 1$ \\
\hline $\mathrm{X}-28$ & Bu544 & 14.56 & 0.77 & 1.58 & 2.08 & 2.20 & 2,4 & 4 & 53600 & $35 / 1$ \\
\hline II-12 & Bu31 & 14.58 & 0.75 & 1.62 & 2.09 & 2.18 & 2,4 & 8 & 106300 & $50 / 1$ \\
\hline XI-38 & Bu512 & 14.58 & 0.75 & 1.60 & 2.07 & 2.16 & 2,4 & 4 & 53600 & $35 / 1$ \\
\hline IX-6 & $\ldots$ & 14.61 & 0.81 & $\ldots$ & $\ldots$ & $\ldots$ & 3 & 8 & 106300 & $60 / 1$ \\
\hline$X-3$ & $\ldots$ & 14.63 & 0.79 & $\ldots$ & $\ldots$ & $\ldots$ & 3 & 8 & 106300 & $65 / 1$ \\
\hline IX-10 & $\ldots$ & 14.63 & 0.77 & $\ldots$ & $\ldots$ & $\ldots$ & 3 & 8 & 106300 & $60 / 1$ \\
\hline IX-2 & $\ldots$ & 14.72 & 0.72 & $\ldots$ & $\ldots$ & $\ldots$ & 3 & 8 & 106300 & $60 / 1$ \\
\hline$\ldots$ & Bu486 & 14.74 & 0.76 & 1.65 & 2.17 & 2.24 & 2,4 & 8 & 106300 & $60 / 1$ \\
\hline $\mathrm{I}-40$ & $\mathrm{Bu} 254$ & 14.77 & 0.78 & 1.51 & 1.99 & 2.13 & 2,4 & 8 & 106300 & $35 / 1$ \\
\hline XII-5 & Bu396 & 14.77 & 0.75 & 1.54 & 2.09 & 2.11 & 2,4 & 4 & 53600 & $30 / 1$ \\
\hline III-11 & Bu27 & 15.16 & 0.76 & 1.52 & 1.97 & 2.02 & 2,4 & 8 & 106300 & $35 / 1$ \\
\hline V-84 & Bu183 & 15.68 & 0.71 & 1.49 & 1.94 & 2.01 & 2,4 & 8 & 106300 & $30 / 1$ \\
\hline
\end{tabular}

References. (1) Sandage \& Walker 1966; (2) Buonanno et al. 1983; (3) Rees 1992; (4) 2MASS.

co-addition, order extraction, wavelength calibration, sky subtraction, radial velocity (RV) cross-correlation, and continuum normalization of the spectra. Scattered light was not removed because we found that this produced negative counts in the cores of the $\mathrm{Ca}$ II $\mathrm{H}$ and $\mathrm{K}$ lines. Each exposure consists of 3-4 sub-exposures (of length 3600-3900 s) co-added to facilitate removal of cosmic rays.

\section{ANALYSIS}

\subsection{Radial Velocities}

We measure the RV of each exposure of each star by crosscorrelating against a template. We produce this template by measuring wavelengths of individual spectral lines in the best exposure of the brightest star (VII-18) and shifting this spectrum to zero RV. The template zeropoint has a precision of about $0.6 \mathrm{~km} \mathrm{~s}^{-1}$. The mean RV and standard deviation for each star are listed in Table 3. Heliocentric corrections are computed with the IRAF task rvcorrect. The mean (heliocentric) RV is $-119.7 \pm 0.8(\sigma=4.8) \mathrm{km} \mathrm{s}^{-1}$.

No telluric lines are covered in any of these spectra, so we cannot assess the absolute zeropoint of the RV measurements, but the mean RV is in good agreement with previous studies. Drukier et al. (2007) observed all of the M92 stars in our sample, and we find a mean offset of $2.1 \pm 0.3 \mathrm{~km} \mathrm{~s}^{-1}$ (our study minus theirs). Mészáros et al. (2009) observed 30 M92 stars in common with us, and we find a mean offset of $2.1 \pm 0.4 \mathrm{~km} \mathrm{~s}^{-1}$. Soderberg et al. (1999) and Pilachowski et al. (2000) measured the RV of 19 and 23 stars in common with our sample, and we find an offset of $1.4 \pm 0.3 \mathrm{~km} \mathrm{~s}^{-1}$ (note that Pilachowski et al. normalized their RV measurements to those of Soderberg et al.). Shetrone et al. (2001) report the RV of one star in common, VII-18, which is different from our measurement by $1 \mathrm{~km} \mathrm{~s}^{-1}$. The observed velocity dispersion that we derive from 39 stars in M92, $\sigma=4.8 \pm 0.8$, also compares well with previous estimates $\left(5.0 \pm 0.5 \mathrm{~km} \mathrm{~s}^{-1}, 49\right.$ stars, Rees 1992 and Pryor \& Meylan 1993, reanalyzing the unpublished data of Lupton et al. 1985; 
Table 3

Radial Velocities and Stellar Parameters

\begin{tabular}{|c|c|c|c|c|c|c|c|}
\hline Star Name & $\begin{array}{c}V_{\mathrm{rad}} \\
\left(\mathrm{km} \mathrm{s}^{-1}\right)\end{array}$ & $\begin{array}{c}\sigma \\
\left(\mathrm{km} \mathrm{s}^{-1}\right)\end{array}$ & $\begin{array}{l}T_{\text {eff }} \\
(\mathrm{K})\end{array}$ & $\log g$ & $\begin{array}{c}v_{t} \\
\left(\mathrm{~km} \mathrm{~s}^{-1}\right)\end{array}$ & {$[\mathrm{M} / \mathrm{H}]$} & Notes \\
\hline VII-18 & -116.6 & 0.3 & 4300 & 0.60 & 2.60 & -2.4 & \\
\hline XII-8 & -117.0 & 0.8 & 4450 & 1.00 & 2.40 & -2.4 & \\
\hline$V-45$ & -118.2 & 0.5 & 4440 & 1.00 & 2.35 & -2.4 & \\
\hline XI-19 & -115.1 & 0.6 & 4500 & 1.05 & 2.40 & -2.4 & \\
\hline XI-80 & -122.7 & 0.5 & 4470 & 1.10 & 2.35 & -2.4 & \\
\hline XII-34 & -113.6 & 0.5 & 4670 & 1.40 & 2.30 & -2.4 & \\
\hline IV-10 & -116.4 & 1.3 & 4570 & 1.35 & 2.25 & -2.4 & \\
\hline IV-79 & -118.3 & 1.0 & 4560 & 1.35 & 2.20 & -2.4 & \\
\hline VI-7 & -137.8 & 0.4 & $\ldots$ & $\ldots$ & $\ldots$ & $\ldots$ & Non-member \\
\hline IV-2 & -122.9 & 0.3 & 4640 & 1.40 & 2.25 & -2.4 & \\
\hline VII-10 & -118.1 & 0.9 & 4680 & 1.45 & 2.25 & -2.4 & RHB/AGB \\
\hline VI-18 & -123.3 & 0.8 & 4730 & 1.55 & 2.25 & -2.4 & RHB/AGB \\
\hline IX-49 & -126.7 & 0.9 & 4720 & 1.60 & 2.20 & -2.4 & \\
\hline IV-40 & -117.3 & 1.0 & 4760 & 1.60 & 2.20 & -2.4 & RHB/AGB \\
\hline XII-31 & -113.6 & 1.3 & 4720 & 1.65 & 2.15 & -2.4 & RHB/AGB \\
\hline VIII-44 & -117.1 & 0.6 & 4750 & 1.70 & 2.15 & -2.4 & \\
\hline XI-10 & -127.0 & 0.6 & 4780 & 1.70 & 2.15 & -2.4 & \\
\hline VIII-24 & -116.2 & 0.4 & 4900 & 1.80 & 2.25 & -2.4 & RHB/AGB \\
\hline III-4 & -118.4 & 0.9 & 5000 & 1.85 & 2.30 & -2.4 & RHB/AGB \\
\hline Bu166 & -124.2 & 0.9 & 4730 & 1.70 & 2.10 & -2.4 & \\
\hline IX-89 & -115.0 & 0.5 & 4780 & 1.75 & 2.10 & -2.4 & \\
\hline VII-79 & -114.1 & 0.5 & 4760 & 1.75 & 2.10 & -2.4 & RHB/AGB \\
\hline II-39 & -118.8 & 0.7 & 4880 & 1.85 & 2.15 & -2.4 & \\
\hline VII-68 & -115.9 & 1.3 & 4740 & 1.75 & 2.05 & -2.4 & RHB/AGB \\
\hline XII-18 & -123.0 & 0.8 & 5140 & 2.00 & 2.30 & -2.4 & RHB/AGB \\
\hline V-78 & -123.3 & 0.9 & 5080 & 2.00 & 2.25 & -2.4 & RHB/AGB \\
\hline II-24 & -118.3 & 1.3 & 4910 & 1.95 & 2.10 & -2.4 & \\
\hline $\mathrm{X}-28$ & -116.3 & 0.7 & 4970 & 2.00 & 2.15 & -2.4 & \\
\hline II-12 & -117.6 & 1.1 & 4960 & 1.95 & 2.10 & -2.4 & \\
\hline XI-38 & -112.8 & 1.5 & 4980 & 2.00 & 2.15 & -2.4 & \\
\hline IX-6 & -123.3 & 1.4 & 4930 & 2.00 & 2.05 & -2.4 & \\
\hline$X-3$ & -123.1 & 0.5 & 4940 & 2.00 & 2.10 & -2.4 & \\
\hline IX-10 & -121.6 & 1.2 & 4940 & 2.00 & 2.10 & -2.4 & \\
\hline IX-2 & -123.3 & 0.9 & 4960 & 2.05 & 2.05 & -2.4 & \\
\hline Bu486 & -121.4 & 0.9 & 4890 & 2.00 & 2.00 & -2.4 & \\
\hline $\mathrm{I}-40$ & -122.0 & 1.1 & 5070 & 2.10 & 2.15 & -2.4 & \\
\hline XII-5 & -116.9 & 0.5 & 5030 & 2.10 & 2.10 & -2.4 & \\
\hline III-11 & -117.6 & 1.5 & 5120 & 2.30 & 2.05 & -2.4 & \\
\hline V-84 & -122.1 & 1.5 & 5150 & 2.50 & 1.90 & -2.4 & \\
\hline
\end{tabular}

$3.3 \pm 0.5 \mathrm{~km} \mathrm{~s}^{-1}, 35$ stars, Soderberg et al. 1999; $4.4 \pm$ $0.6 \mathrm{~km} \mathrm{~s}^{-1}, 61 \mathrm{stars}$, Pilachowski et al. 2000; $4.8 \pm 0.4 \mathrm{~km} \mathrm{~s}^{-1}$, 64 stars, Mészáros et al. 2009; $5.1 \pm 2.4 \mathrm{~km} \mathrm{~s}^{-1}, 5$ stars, Cohen \& McCarthy 1997).

Mészáros et al. (2009) reported one RV variable star among the stars in our sample, XI-38, which is confirmed by our measurements. No other stars exhibit any significant $(\gtrsim 3 \sigma) \mathrm{RV}$ drift over the 14 years that span the four sets of observations. ${ }^{6}$

In summary, our mean RV, individual stellar RVs, and observed velocity dispersion are all in reasonably good agreement with previous measurements.

\footnotetext{
6 There has been considerable uncertainty surrounding the membership of star VI-7 (= ZDA1 and ZNG4). This star appears to be RV variable, with velocities ranging from -90 to $-158 \mathrm{~km} \mathrm{~s}^{-1}$ (Strom \& Strom 1971; Zinn 1973; Norris \& Zinn 1977; Pilachowski et al. 2000; this study). Proper motion studies have assigned various probabilities to its membership: 15\% (Cudworth 1976), 99\% (Rees 1992), and 68\% (Tucholke et al. 1996). Carbon et al. (1982) also considered VI-7 a non-member based on the available RV and proper motion data and on account of its stronger $\mathrm{Ca}$ II $\mathrm{H}$ and $\mathrm{K}$ lines. Our abundance analysis finds $[\mathrm{Fe} / \mathrm{H}]=-2.0$, thus quantifying the Carbon et al. assertion and further strengthening this conclusion.
}

\subsection{Model Atmosphere Parameters}

Our spectra cover a very narrow wavelength range, and this naturally restricts the number of methods available to determine model atmosphere parameters. Effective temperatures $\left(T_{\text {eff }}\right)$ calculated from broadband color- $T_{\text {eff }}$ relations provide a satisfactory option. The sensitivity of the $B$ band to individual stellar $\mathrm{CN}$ and $\mathrm{CH}$ band strengths makes the $B-V$ color- $T_{\text {eff }}$ relation an undesirable option if alternatives exist. We use $J H K$ broadband photometry from 2MASS, available for most of our sample, to calculate temperatures from the $V-J, V-H$, and $V-K$ color $-T_{\text {eff }}$ relations. We average the temperatures predicted from these three colors as given by the metallicitycalibrated relations for giants presented in Ramírez \& Meléndez (2005b). These temperatures are listed in Table 3 . An uncertainty of $\Delta V=0.05 \mathrm{mag}$ translates to changes in the $T_{\text {eff }}$ predicted from $V-K$ of $50 \mathrm{~K}$. Standard deviations of the residuals after applying a linear least-squares fit to each relation are each 50$60 \mathrm{~K}$. We then interpolate temperatures for stars lacking 2MASS photometry from these relations.

Since the distance to M92 is well known, we calculate surface gravities according to the relation

$$
\begin{aligned}
\log g= & 0.4\left(M_{K, \star}+\mathrm{BC}_{K}-M_{\mathrm{bol}, \odot}\right)+\log g_{\odot} \\
& +4 \log \left(T_{\mathrm{eff}, \star} / T_{\mathrm{eff}, \odot}\right)+\log \left(m_{\star} / m_{\odot}\right),
\end{aligned}
$$

where a star's apparent magnitude is related to its absolute magnitude through the distance modulus and bolometric correction (BC). We transform $K$ magnitudes from the 2MASS system to the TCS system according to Equation (5c) of Ramírez \& Meléndez (2005a) and interpolate (in $V-K$ and $[\mathrm{Fe} / \mathrm{H}]$ ) the grid of BCs presented by Alonso et al. (1999). We adopt the M92 distance modulus and reddening listed in Table 1, extinction coefficients given by McCall (2004), $0.8 M_{\odot}$ as the mass of stars on the RGB, and the solar values $M_{\mathrm{bol}, \odot}=4.74, \log g_{\odot}=4.44$, and $T_{\text {eff }, \odot}=5780 \mathrm{~K}$. Again, since not all stars have 2MASS photometry, we interpolate surface gravity from the relationship between $V$ and $\log g$ established by those stars that do. The scatter in this relationship is only 0.04 dex. Final $\log g$ values are listed in Table 3. They are relatively insensitive to uncertainties in the input parameters. For $\Delta m_{K}, \Delta m-K$, or $\Delta \mathrm{BC}=$ $\pm 0.1, \Delta \log g=\mp 0.04$; for $\Delta T_{\text {eff }}= \pm 60 \mathrm{~K}, \Delta \log g=$ $\mp 0.02$; and for $\Delta m_{\star}= \pm 0.15 M_{\odot}, \Delta \log g={ }_{-0.08}^{+0.09}$. Several tenths of a solar mass may be lost between the time a star leaves the main sequence and arrives on the red horizontal branch (RHB; e.g., Preston et al. 2006), but this effect has little impact on the relative surface gravities calculated here.

We calculate microturbulent velocities $\left(v_{t}\right)$ from the empirical relationship between $T_{\text {eff }}, \log g$, and $v_{t}$ for metal-poor field giants derived by Gratton et al. (1996). These results are in good agreement with previous spectroscopically derived estimates of $v_{t}$ for red giants in M92 (Shetrone 1996; Shetrone et al. 2001; Johnson 2002), including earlier studies that found $2 \mathrm{~km} \mathrm{~s}^{-1}$ was an adequate estimate for all stars (Sneden et al. 1991, 2000). The mean difference between the empirical relationship and the latter approach is only $\Delta v_{t}=0.18 \pm 0.02(\sigma=0.15) \mathrm{km} \mathrm{s}^{-1}$ (in the sense of the Gratton et al. relation-2.0). These uncertainties are well within the precision regularly achieved for analyses of metal-poor giants.

Sneden et al. (2000) obtained spectra covering $250 \AA$ near $5900 \AA$ for 34 stars in M92 using Hydra. That study computed $T_{\text {eff }}$ and $\log g$ by comparing dereddened $B-V$ and $M_{V}$ with the predicted colors and magnitudes derived from model atmospheres (Carbon et al. 1982). For the 18 stars in common 
with Sneden et al., we find mean differences of $\Delta T_{\text {eff }}=-48 \pm$ $16(\sigma=67) \mathrm{K}$ and $\Delta \log g=-0.16 \pm 0.03(\sigma=0.15)$. There are no significant trends with either $T_{\text {eff }}$ or $\log g$.

Finally, we uniformly adopt a metallicity of $[\mathrm{M} / \mathrm{H}]=$ -2.4 for all model atmospheres. We generate model atmospheres from the MARCS grid of one-dimensional, spherical, standard composition (i.e., $\alpha$-enhanced at this metallicity) models computed assuming local thermodynamic equilibrium (LTE; Gustafsson et al. 2008) using interpolation software kindly provided by A. McWilliam (2009, private communication). We emphasize that our primary goal is to examine the dispersion in the abundance ratios, so the absolute temperature and metallicity scales are only of secondary importance.

\subsection{Derivation of Abundances}

The resolution of our spectra is considerably lower than that commonly used for detailed abundance analyses, and the S/N is generally a decreasing function of luminosity along the RGB. As such, we perform a differential abundance analysis to search for star-to-star chemical dispersion among M92 red giants. Only 19 stars in our sample have $\mathrm{S} / \mathrm{Ns}$ sufficient to derive reliable abundances. The differential abundances are then placed on an absolute scale by computing abundances in one star by the usual techniques. Here we describe these methods in more detail.

We adopt XII-8 as our abundance reference because its $\mathrm{S} / \mathrm{N}$ is among the highest we have attained, 100/1 at $4000 \AA$. Nearly all absorption lines are blended at our spectral resolution, so we derive abundances in XII- 8 by spectrum synthesis. We can reliably derive abundances for 21 species of 19 elements in this star (C, N, Si I, Sc I, Sc II, Ti I, Ti II, V II, Cr I, Mn I, Fe I, Co I, Ni I, Y II, Zr II, La II, Ce II, Nd II, Eu II, Ho II, and Er II). Our line list is given in Table 4. We use the latest version (2010) of the spectrum analysis code MOOG (Sneden 1973) to generate synthetic spectra. Abundances derived for each line in XII-8 are listed in Table 5.

Next, for each line in each star we calculate differential abundances relative to the corresponding line in XII-8. The spectrum generated by MOOG for each line is divided by a synthetic spectrum of that line in XII-8. This quotient is compared to the quotient of the observed spectra of the two stars. Uncertainties are computed according to $\chi^{2}$ statistics regarding the goodness of fit between the observed and synthetic spectra ratios.

This technique yields a differential abundance and relative uncertainty for each line in each star. These values are reported in Table 6 . We find that $\mathrm{S} / \mathrm{N} \gtrsim 65$ is generally necessary to derive reliable differential abundances of the $n$-capture elements, though the minimum $\mathrm{S} / \mathrm{N}$ varies slightly. Final abundances are computed by performing a weighted average of the differentials for a given species and adding this mean differential to the mean $\log \epsilon$ value for that species derived in XII-8. S.S. abundances used to compute the $[\mathrm{X} / \mathrm{Fe}]$ ratios are taken from Asplund et al. (2009). Tables 7-13 list, for each of the 19 stars, $\log \epsilon$ abundances (Column 1), [X/Fe] ratios (Column 2), standard error $(\sigma / \sqrt{N}$, Column 3), standard deviation ( $\sigma$, Column 4), and number of lines used ( $N$, Column 5$)$.

We stress that the uncertainties $(1 \sigma)$ are computed with respect to the differentials; thus, they reflect internal uncertainties only. In the results and discussion that follows, we reference these uncertainties unless otherwise noted. Absolute uncertainties may be computed by combining the standard error of the species from the star in question with that of XII- 8 and the uncertainty associated with the atmospheric parameters.
Table 4

Atomic Data

\begin{tabular}{|c|c|c|c|c|c|}
\hline Species & $Z$ & Wavelength (A) & E.P. $(\mathrm{eV})$ & $\log (g f)$ & Reference \\
\hline $\mathrm{C}\left({ }^{12} \mathrm{CH}\right)$ & 6 & $4000.98+4001.07$ & 0.64 & $-1.12,-1.10$ & 1 \\
\hline $\mathrm{C}\left({ }^{12} \mathrm{CH}\right)$ & 6 & $4020.02+4020.18$ & 0.46 & $-1.38,-1.35$ & 1 \\
\hline $\mathrm{N}(\mathrm{CN})$ & 7 & $3879.0-3883.5$ & $\ldots$ & $\ldots$ & 2 \\
\hline Si I & 14 & 3905.52 & 1.91 & -1.04 & 3 \\
\hline Sc I & 21 & 3911.82 & 0.02 & +0.40 & 2 \\
\hline Sc II & 21 & $3989.13^{\mathrm{a}}$ & 0.32 & -2.72 & 2,4 \\
\hline Ti I & 22 & 3904.78 & 0.90 & +0.03 & 2 \\
\hline Ti I & 22 & 3989.76 & 0.02 & -0.13 & 5,6 \\
\hline Ti I & 22 & 3998.64 & 0.05 & +0.01 & 5,6 \\
\hline Ti I & 22 & 4008.93 & 0.02 & -1.02 & 5,6 \\
\hline Ti II & 22 & 3987.61 & 0.61 & -2.93 & 7 \\
\hline Ti II & 22 & 4025.13 & 0.61 & -2.14 & 7 \\
\hline V II & 23 & 3951.96 & 1.48 & -0.78 & 8 \\
\hline V II & 23 & 4002.94 & 1.43 & -1.45 & 8 \\
\hline V II & 23 & 4005.71 & 1.82 & -0.52 & 8 \\
\hline V II & 23 & 4023.38 & 1.80 & -0.69 & 8 \\
\hline V II & 23 & 4036.78 & 1.48 & -1.59 & 8 \\
\hline Cr I & 24 & 3908.76 & 1.00 & -1.05 & 9 \\
\hline Mn I & 25 & 4018.10 & 2.11 & -0.19 & 10 \\
\hline Mn I & 25 & 4030.75 & 0.00 & -0.47 & 11 \\
\hline Mn I & 25 & 4033.06 & 0.00 & -0.62 & 11 \\
\hline Mn I & 25 & 4034.48 & 0.00 & -0.81 & 11 \\
\hline Mn I & 25 & 4041.36 & 2.11 & +0.28 & 11 \\
\hline $\mathrm{Fe}_{\mathrm{I}}$ & 26 & 3891.93 & 3.41 & -0.73 & 12 \\
\hline $\mathrm{Fe}_{\mathrm{I}}$ & 26 & 3899.03 & 2.45 & -1.81 & 12 \\
\hline $\mathrm{Fe}_{\mathrm{I}}$ & 26 & 3985.39 & 3.30 & -0.99 & 12 \\
\hline $\mathrm{Fe}_{\mathrm{I}}$ & 26 & 4001.66 & 2.17 & -1.90 & 12 \\
\hline $\mathrm{Fe} I$ & 26 & 4007.27 & 2.76 & -1.28 & 12 \\
\hline $\mathrm{Fe}_{\mathrm{I}}$ & 26 & 4013.82 & 3.02 & -1.70 & 2 \\
\hline $\mathrm{Fe}_{\mathrm{I}}$ & 26 & $4017.08+4017.15$ & $2.76,3.05$ & $-1.99,-1.06$ & 2,12 \\
\hline $\mathrm{Fe} I$ & 26 & $4032.45+4032.63$ & $4.26,1.48$ & $-0.84,-2.38$ & 2,12 \\
\hline $\mathrm{Fe} I$ & 26 & 4044.61 & 2.83 & -1.22 & 12 \\
\hline Co I & 27 & 3995.31 & 0.92 & -0.14 & 13 \\
\hline Co I & 27 & 4020.90 & 0.43 & -2.04 & 13 \\
\hline Co I & 27 & 4027.02 & 0.17 & -2.87 & 2 \\
\hline Ni I & 28 & 3912.97 & 0.02 & -3.70 & 2 \\
\hline Y II & 39 & 3950.36 & 0.10 & -0.49 & 14 \\
\hline Y II & 39 & 3982.60 & 0.13 & -0.49 & 14 \\
\hline Zr II & 40 & 3991.13 & 0.76 & -0.23 & 15 \\
\hline Zr II & 40 & 4029.68 & 0.71 & -0.74 & 15 \\
\hline Zr II & 40 & 4050.33 & 0.71 & -1.00 & 15 \\
\hline La II & 57 & $3949.10^{\mathrm{a}}$ & 0.40 & +0.49 & 16 \\
\hline La II & 57 & $3988.51^{\mathrm{a}}$ & 0.40 & +0.21 & 16 \\
\hline La II & 57 & $3995.74^{\mathrm{a}}$ & 0.17 & -0.06 & 16 \\
\hline La II & 57 & $4031.69^{a}$ & 0.32 & -0.08 & 16 \\
\hline Ce II & 58 & 4042.58 & 0.50 & +0.00 & 17 \\
\hline $\mathrm{Nd}$ II & 60 & 4023.00 & 0.56 & +0.04 & 18 \\
\hline $\mathrm{Nd}$ II & 60 & 4051.14 & 0.38 & -0.30 & 18 \\
\hline Eu II & 63 & $3907.11^{\mathrm{a}}$ & 0.21 & +0.17 & 19 \\
\hline Ho II & 67 & $3891.00^{\mathrm{a}}$ & 0.08 & +0.46 & 20 \\
\hline Er II & 68 & 3896.23 & 0.06 & -0.12 & 21 \\
\hline
\end{tabular}

Note. ${ }^{a}$ Includes hyperfine splitting structure.

References. (1) B. Plez 2007, private communication; (2) Kurucz \& Bell 1995; (3) O’Brian \& Lawler 1991; (4) Lawler \& Dakin 1989; (5) Blackwell et al. 1982; (6) Grevesse et al. 1989; (7) Pickering et al. 2002; (8) Biémont et al. 1989; (9) Sobeck et al. 2007; (10) Blackwell-Whitehead \& Bergemann 2007; (11) Booth et al. 1984; (12) O’Brian et al. 1991; (13) Nitz et al. 1999; (14) Hannaford et al. 1982; (15) Malcheva et al. 2006; (16) Lawler et al. 2001a; (17) Lawler et al. 2009; (18) Den Hartog et al. 2003; (19) Lawler et al. 2001b; (20) Lawler et al. 2004; (21) Lawler et al. 2008.

The magnitude of this final source of uncertainty is assessed by rederiving the abundance ratios of several key elements in XII-8 
Table 5

Abundances in Reference Star XII-8

\begin{tabular}{|c|c|c|c|}
\hline Species & $Z$ & Wavelength $(\AA)$ & $\log \epsilon$ \\
\hline $\mathrm{C}\left({ }^{12} \mathrm{CH}\right.$ & 6 & 4001.03 & 5.05 \\
\hline $\mathrm{C}\left({ }^{12} \mathrm{CH}\right)$ & 6 & 4020.10 & 5.10 \\
\hline $\mathrm{N}(\mathrm{CN})$ & 7 & 3883.00 & 5.65 \\
\hline Si I & 14 & 3905.52 & 5.05 \\
\hline Sc I & 21 & 3911.82 & 0.40 \\
\hline Sc II & 21 & 3989.13 & 0.80 \\
\hline Ti I & 22 & 3904.78 & 2.50 \\
\hline Ti I & 22 & 3989.76 & 1.88 \\
\hline Ti I & 22 & 3998.64 & 2.13 \\
\hline Ti I & 22 & 4008.93 & 2.35 \\
\hline Ti II & 22 & 3987.61 & 2.95 \\
\hline Ti II & 22 & 4025.13 & 2.91 \\
\hline $\mathrm{V}_{\text {II }}$ & 23 & 3951.96 & 1.29 \\
\hline V II & 23 & 4002.94 & 1.64 \\
\hline $\mathrm{V}_{\text {II }}$ & 23 & 4005.71 & 1.91 \\
\hline V II & 23 & 4023.38 & 1.76 \\
\hline $\mathrm{V}_{\mathrm{II}}$ & 23 & 4036.78 & 1.60 \\
\hline CrI & 24 & 3908.76 & 2.20 \\
\hline Mn I & 25 & 4018.10 & 2.08 \\
\hline Mn I & 25 & 4030.75 & 2.00 \\
\hline Mn I & 25 & 4033.06 & 1.90 \\
\hline Mn I & 25 & 4034.48 & 1.80 \\
\hline Mn I & 25 & 4041.36 & 2.05 \\
\hline $\mathrm{Fe}_{\mathrm{I}}$ & 26 & 3891.93 & 4.71 \\
\hline $\mathrm{Fe} I$ & 26 & 3899.03 & 4.44 \\
\hline $\mathrm{Fe}_{\mathrm{I}}$ & 26 & 3985.39 & 4.29 \\
\hline $\mathrm{Fe}_{\mathrm{I}}$ & 26 & 4001.66 & 4.72 \\
\hline $\mathrm{Fe}_{\mathrm{I}}$ & 26 & 4007.27 & 4.53 \\
\hline $\mathrm{Fe}_{\mathrm{I}}$ & 26 & 4013.82 & 4.90 \\
\hline $\mathrm{Fe}_{\mathrm{I}}$ & 26 & 4017.15 & 4.64 \\
\hline $\mathrm{Fe} I$ & 26 & 4032.63 & 4.64 \\
\hline $\mathrm{Fe}_{\mathrm{I}}$ & 26 & 4044.61 & 4.64 \\
\hline Co I & 27 & 3995.31 & 1.77 \\
\hline Co I & 27 & 4020.90 & 2.37 \\
\hline Co I & 27 & 4027.02 & 2.50 \\
\hline Ni I & 28 & 3912.97 & 3.25 \\
\hline$Y_{\text {II }}$ & 39 & 3950.36 & -0.60 \\
\hline $\mathrm{Y}_{\text {II }}$ & 39 & 3982.60 & -1.00 \\
\hline Zr II & 40 & 3991.13 & 0.03 \\
\hline Zr II & 40 & 4029.68 & 0.59 \\
\hline Zr II & 40 & 4050.33 & 0.20 \\
\hline La II & 57 & 3949.10 & -1.30 \\
\hline La II & 57 & 3988.51 & -1.40 \\
\hline La II & 57 & 3995.74 & -1.45 \\
\hline La II & 57 & 4031.69 & -1.35 \\
\hline Ce II & 58 & 4042.58 & -0.85 \\
\hline $\mathrm{Nd}$ II & 60 & 4023.00 & -0.85 \\
\hline $\mathrm{Nd}$ II & 60 & 4051.14 & -1.05 \\
\hline Eu II & 63 & 3907.11 & -1.75 \\
\hline Ho II & 67 & 3891.00 & -1.85 \\
\hline Er II & 68 & 3896.23 & -1.30 \\
\hline
\end{tabular}

after making reasonable variations to the model atmosphere parameters. These values are listed in Table 14.

\subsection{Comments on Individual Species}

A few comments regarding these abundances are warranted. Figure 2 demonstrates that our analysis produces an artificial relation between $[\mathrm{Fe} / \mathrm{H}]$ and $T_{\text {eff }}$. Our $\mathrm{Fe}$ abundances span a range from $-2.89<[\mathrm{Fe} / \mathrm{H}]<-2.35$ with a mean of $[\mathrm{Fe} / \mathrm{H}]=-2.70 \pm 0.03(\sigma=0.14)$. This mean metallicity is lower than has been derived in previous studies, and we

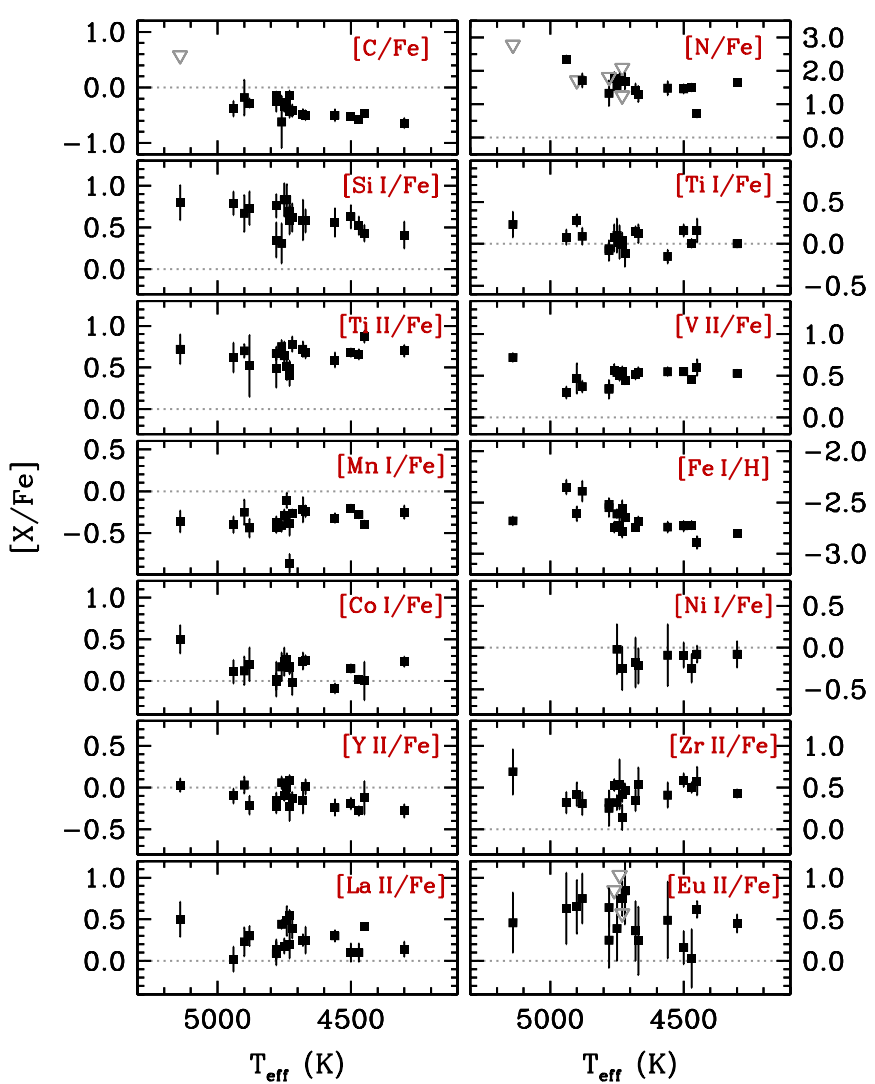

Figure 2. Abundance ratios as a function of $T_{\text {eff. }}$. Detections are indicated by filled squares, and upper limits are indicated by downward-facing open triangles. Dotted lines represent the S.S. ratios. Species with limited numbers of detections are omitted. Only the internal (i.e., star-to-star) uncertainties are shown. (A color version of this figure is available in the online journal.)

Table 6

Line-by-Line Abundances

\begin{tabular}{lccrc}
\hline \hline Species & $Z$ & Wavelength $(\AA)$ & $\Delta($ dex $)$ & $\sigma($ dex $)$ \\
\hline \multicolumn{5}{c}{ VII-18 } \\
\hline C $\left({ }^{12} \mathrm{CH}\right)$ & 6 & 4001.03 & -0.18 & 0.12 \\
C $\left({ }^{12} \mathrm{CH}\right)$ & 6 & 4020.10 & 0.00 & 0.12 \\
N $(\mathrm{CN})$ & 7 & 3883.00 & 1.03 & 0.10 \\
Si I & 14 & 3905.52 & 0.07 & 0.16 \\
Sc I & 21 & 3911.82 & 0.12 & 0.17 \\
Sc II & 21 & 3989.13 & 0.28 & 0.12 \\
Ti I & 22 & 3904.78 & -0.02 & 0.16 \\
Ti I & 22 & 3989.76 & -0.11 & 0.10 \\
Ti I & 22 & 3998.64 & 0.04 & 0.21 \\
Ti I & 22 & 4008.93 & -0.09 & 0.08 \\
\hline
\end{tabular}

Notes. The data for one example star are shown here.

(This table is available in its entirety in machine-readable and Virtual Observatory (VO) forms in the online journal. A portion is shown here for guidance regarding its form and content.)

address this point in detail in the Appendix. We have detected no lines of Fe II, the dominant Fe species in these stars, in our spectra; however, many of the elements we are interested in studying are only detected in the singly ionized state. This casts considerable uncertainty on the accuracy of $[\mathrm{X} / \mathrm{Fe}]$ ratios when $\mathrm{X}$ is a singly ionized species (e.g., [La II/Fe I] or [Eu II/Fe I]), but ratios among species of the same ionization state should be more robust. 
Table 7

Stellar Abundances I

\begin{tabular}{|c|c|c|c|c|c|c|c|c|c|c|c|c|c|c|c|c|}
\hline \multirow[t]{2}{*}{ Species } & \multirow[t]{2}{*}{$Z$} & \multicolumn{5}{|c|}{ II-39 } & \multicolumn{5}{|c|}{ IV-40 } & \multicolumn{5}{|c|}{ IV-79 } \\
\hline & & $\log \epsilon$ & {$[\mathrm{X} / \mathrm{Fe}]$} & $\sigma_{\mu}$ & $\sigma$ & $N$ & $\log \epsilon$ & {$[\mathrm{X} / \mathrm{Fe}]$} & $\sigma_{\mu}$ & $\sigma$ & $N$ & $\log \epsilon$ & {$[\mathrm{X} / \mathrm{Fe}]$} & $\sigma_{\mu}$ & $\sigma$ & $N$ \\
\hline $\mathrm{C}\left({ }^{12} \mathrm{CH}\right)$ & 6 & 5.76 & -0.28 & 0.08 & 0.12 & 2 & 5.07 & -0.62 & 0.47 & 0.47 & 1 & 5.19 & -0.50 & 0.11 & 0.16 & 2 \\
\hline $\mathrm{N}(\mathrm{CN})$ & 7 & 7.15 & 1.71 & 0.21 & 0.21 & 1 & 6.85 & 1.76 & 0.20 & 0.20 & 1 & 6.57 & 1.48 & 0.21 & 0.21 & 1 \\
\hline Si I & 14 & 5.85 & 0.73 & 0.20 & 0.20 & 1 & 5.08 & 0.31 & 0.24 & 0.24 & 1 & 5.33 & 0.56 & 0.17 & 0.17 & 1 \\
\hline Sc I & 21 & $\ldots$ & $\ldots$ & $\ldots$ & $\ldots$ & $\ldots$ & $\ldots$ & $\ldots$ & $\ldots$ & $\ldots$ & $\ldots$ & $\ldots$ & $\ldots$ & $\ldots$ & $\ldots$ & $\ldots$ \\
\hline Sc II & 21 & $\ldots$ & $\ldots$ & $\ldots$ & $\ldots$ & $\ldots$ & $\ldots$ & $\ldots$ & $\ldots$ & $\ldots$ & $\ldots$ & 1.11 & 0.70 & 0.28 & 0.28 & 1 \\
\hline Ti I & 22 & 2.65 & 0.09 & 0.10 & 0.20 & 4 & 2.28 & 0.07 & 0.16 & 0.28 & 3 & 2.07 & -0.14 & 0.09 & 0.18 & 4 \\
\hline Ti II & 22 & 3.08 & 0.52 & 0.37 & 0.52 & 2 & 2.96 & 0.74 & 0.08 & 0.12 & 2 & 2.80 & 0.59 & 0.09 & 0.13 & 2 \\
\hline V II & 23 & 1.91 & 0.38 & 0.07 & 0.15 & 5 & 1.76 & 0.57 & 0.07 & 0.15 & 4 & 1.74 & 0.55 & 0.05 & 0.11 & 5 \\
\hline Cr I & 24 & $\ldots$ & $\ldots$ & $\ldots$ & $\ldots$ & $\ldots$ & $\ldots$ & $\ldots$ & $\ldots$ & $\ldots$ & $\ldots$ & $\ldots$ & $\ldots$ & $\ldots$ & $\ldots$ & $\ldots$ \\
\hline Mn I & 25 & 2.60 & -0.43 & 0.11 & 0.22 & 4 & 2.28 & -0.41 & 0.07 & 0.14 & 4 & 2.37 & -0.33 & 0.06 & 0.12 & 4 \\
\hline $\mathrm{Fe}_{\mathrm{I}}$ & 26 & 5.11 & -2.39 & 0.10 & 0.28 & 8 & 4.76 & -2.74 & 0.05 & 0.13 & 7 & 4.76 & -2.74 & 0.05 & 0.15 & 9 \\
\hline Co I & 27 & 2.80 & 0.20 & 0.20 & 0.20 & 1 & 2.42 & 0.17 & 0.17 & 0.29 & 3 & 2.16 & -0.09 & 0.08 & 0.14 & 3 \\
\hline Ni I & 28 & $\ldots$ & $\ldots$ & $\ldots$ & $\ldots$ & $\ldots$ & $\ldots$ & $\ldots$ & $\ldots$ & $\ldots$ & $\ldots$ & 3.39 & -0.09 & 0.37 & 0.37 & 1 \\
\hline Y II & 39 & -0.39 & -0.20 & 0.11 & 0.16 & 2 & -0.47 & 0.05 & 0.07 & 0.10 & 2 & -0.77 & -0.25 & 0.10 & 0.14 & 2 \\
\hline Zr II & 40 & 0.50 & 0.31 & 0.14 & 0.20 & 2 & 0.37 & 0.52 & 0.07 & 0.12 & 3 & 0.25 & 0.41 & 0.15 & 0.26 & 3 \\
\hline La II & 57 & -0.99 & 0.31 & 0.12 & 0.21 & 3 & -1.20 & 0.44 & 0.05 & 0.09 & 3 & -1.34 & 0.30 & 0.07 & 0.12 & 3 \\
\hline Ce II & 58 & $\ldots$ & $\ldots$ & $\ldots$ & $\ldots$ & $\ldots$ & $\ldots$ & $\ldots$ & $\ldots$ & $\ldots$ & $\ldots$ & $\ldots$ & $\ldots$ & $\ldots$ & $\ldots$ & $\ldots$ \\
\hline Nd II & 60 & $\ldots$ & $\ldots$ & $\ldots$ & $\ldots$ & $\ldots$ & $\ldots$ & $\ldots$ & $\ldots$ & $\ldots$ & $\ldots$ & $\ldots$ & $\ldots$ & $\ldots$ & $\ldots$ & $\ldots$ \\
\hline Eu II & 63 & -1.12 & 0.75 & 0.30 & 0.30 & 1 & $<-1.4$ & $<0.85$ & $\ldots$ & $\ldots$ & $\ldots$ & -1.73 & 0.49 & 0.46 & 0.46 & 1 \\
\hline Ho II & 67 & $\ldots$ & $\ldots$ & $\ldots$ & $\ldots$ & $\ldots$ & $\ldots$ & $\ldots$ & $\ldots$ & $\ldots$ & $\ldots$ & $\ldots$ & $\ldots$ & $\ldots$ & $\ldots$ & $\ldots$ \\
\hline Er II & 68 & $\ldots$ & $\ldots$ & $\ldots$ & $\ldots$ & $\ldots$ & $\ldots$ & $\ldots$ & $\ldots$ & $\ldots$ & $\ldots$ & $\ldots$ & $\ldots$ & $\ldots$ & $\ldots$ & $\ldots$ \\
\hline
\end{tabular}

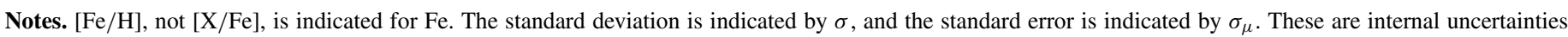
only; see Section 3.3 for a discussion of absolute uncertainties.

Table 8

Stellar Abundances II

\begin{tabular}{|c|c|c|c|c|c|c|c|c|c|c|c|c|c|c|c|c|}
\hline \multirow[t]{2}{*}{ Species } & \multirow[t]{2}{*}{$Z$} & \multicolumn{5}{|c|}{ VI-18 } & \multicolumn{5}{|c|}{ VII-10 } & \multicolumn{5}{|c|}{ VII-18 } \\
\hline & & $\log \epsilon$ & {$[\mathrm{X} / \mathrm{Fe}]$} & $\sigma_{\mu}$ & $\sigma$ & $N$ & $\log \epsilon$ & {$[\mathrm{X} / \mathrm{Fe}]$} & $\sigma_{\mu}$ & $\sigma$ & $N$ & $\log \epsilon$ & {$[\mathrm{X} / \mathrm{Fe}]$} & $\sigma_{\mu}$ & $\sigma$ & $N$ \\
\hline $\mathrm{C}\left({ }^{12} \mathrm{CH}\right)$ & 6 & 5.22 & -0.43 & 0.11 & 0.15 & 2 & 5.21 & -0.48 & 0.09 & 0.13 & 2 & 4.99 & -0.64 & 0.09 & 0.13 & 2 \\
\hline $\mathrm{N}(\mathrm{CN})$ & 7 & $<7.1$ & $<2.08$ & $\ldots$ & $\ldots$ & $\ldots$ & 6.49 & 1.40 & 0.22 & 0.22 & 1 & 6.68 & 1.65 & 0.10 & 0.10 & 1 \\
\hline Si I & 14 & 5.32 & 0.59 & 0.17 & 0.17 & 1 & 5.36 & 0.59 & 0.24 & 0.24 & 1 & 5.12 & 0.41 & 0.16 & 0.16 & 1 \\
\hline Sc I & 21 & $\ldots$ & $\ldots$ & $\ldots$ & $\ldots$ & $\ldots$ & $\ldots$ & $\ldots$ & $\ldots$ & $\ldots$ & $\ldots$ & 0.52 & 0.17 & 0.17 & 0.17 & 1 \\
\hline Sc II & 21 & 0.87 & 0.50 & 0.37 & 0.37 & 1 & $\ldots$ & $\ldots$ & $\ldots$ & $\ldots$ & $\ldots$ & 1.03 & 0.68 & 0.12 & 0.12 & 1 \\
\hline Ti I & 22 & 2.21 & 0.04 & 0.09 & 0.19 & 4 & 2.36 & 0.16 & 0.06 & 0.11 & 3 & 2.14 & 0.00 & 0.04 & 0.08 & 4 \\
\hline Ti II & 22 & 2.57 & 0.41 & 0.12 & 0.17 & 2 & 2.93 & 0.72 & 0.09 & 0.12 & 2 & 2.85 & 0.70 & 0.07 & 0.10 & 2 \\
\hline $\mathrm{V}_{\text {II }}$ & 23 & 1.66 & 0.51 & 0.04 & 0.08 & 4 & 1.71 & 0.53 & 0.06 & 0.13 & 5 & 1.66 & 0.54 & 0.04 & 0.08 & 5 \\
\hline CrI & 24 & $\ldots$ & $\ldots$ & $\ldots$ & $\ldots$ & $\ldots$ & $\ldots$ & $\ldots$ & $\ldots$ & $\ldots$ & $\ldots$ & 2.61 & -0.23 & 0.18 & 0.18 & 1 \\
\hline Mn I & 25 & 1.79 & -0.86 & 0.11 & 0.25 & 5 & 2.47 & -0.22 & 0.15 & 0.33 & 5 & 2.39 & -0.24 & 0.08 & 0.18 & 5 \\
\hline $\mathrm{Fe}_{\mathrm{I}}$ & 26 & 4.72 & -2.78 & 0.06 & 0.18 & 8 & 4.76 & -2.74 & 0.02 & 0.05 & 8 & 4.70 & -2.80 & 0.03 & 0.08 & 9 \\
\hline Co I & 27 & 2.37 & 0.16 & 0.06 & 0.10 & 3 & 2.49 & 0.25 & 0.10 & 0.17 & 3 & 2.42 & 0.23 & 0.06 & 0.11 & 3 \\
\hline $\mathrm{Ni}$ I & 28 & 3.19 & -0.25 & 0.26 & 0.26 & 1 & 3.30 & -0.18 & 0.30 & 0.30 & 1 & 3.34 & -0.08 & 0.16 & 0.16 & 1 \\
\hline Y II & 39 & -0.48 & 0.09 & 0.07 & 0.09 & 2 & -0.68 & -0.15 & 0.16 & 0.22 & 2 & -0.87 & -0.27 & 0.08 & 0.11 & 2 \\
\hline Zr II & 40 & 0.22 & 0.42 & 0.09 & 0.12 & 2 & 0.19 & 0.36 & 0.13 & 0.22 & 3 & 0.21 & 0.43 & 0.05 & 0.09 & 3 \\
\hline La II & 57 & -1.14 & 0.55 & 0.07 & 0.15 & 4 & -1.39 & 0.26 & 0.07 & 0.14 & 4 & -1.57 & 0.14 & 0.10 & 0.19 & 4 \\
\hline Ce II & 58 & $\ldots$ & $\ldots$ & $\ldots$ & $\ldots$ & $\ldots$ & $\ldots$ & $\ldots$ & $\ldots$ & $\ldots$ & $\ldots$ & -0.81 & 0.41 & 0.19 & 0.19 & 1 \\
\hline Nd II & 60 & -0.82 & 0.54 & 0.31 & 0.31 & 1 & $\ldots$ & $\ldots$ & $\ldots$ & $\ldots$ & $\ldots$ & -1.06 & 0.32 & 0.14 & 0.20 & 2 \\
\hline Eu II & 63 & -1.51 & 0.75 & 0.21 & 0.21 & 1 & -1.86 & 0.36 & 0.36 & 0.36 & 1 & -1.83 & 0.45 & 0.11 & 0.11 & 1 \\
\hline Ho II & 67 & -1.31 & 0.99 & 0.14 & 0.14 & 1 & $\ldots$ & $\ldots$ & $\ldots$ & $\ldots$ & $\ldots$ & -2.08 & 0.24 & 0.13 & 0.13 & 1 \\
\hline Er II & 68 & -0.80 & 1.06 & 0.19 & 0.19 & 1 & $\ldots$ & $\ldots$ & $\ldots$ & $\ldots$ & $\ldots$ & -1.22 & 0.66 & 0.16 & 0.16 & 1 \\
\hline
\end{tabular}

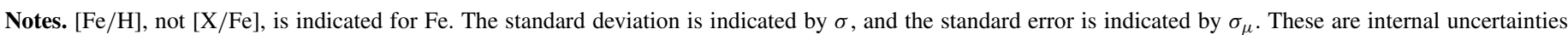
only; see Section 3.3 for a discussion of absolute uncertainties.

The star-to-star dispersion among metal ratios is robust, as illustrated in Figure 2. Our [Ti I/Fe I] ratios are persistently lower than the [Ti II $/ \mathrm{Fe}$ I] ratios by $\sim 0.5$ dex, likely indicating that LTE treatments of the level populations of these neutral species are inadequate (see, e.g., Bergemann 2011 and references therein). This may also account for the [V II $/ \mathrm{Fe} \mathrm{I}]$ ratios that are higher than typically found in metal-poor field stars. If so, we should expect that all $\left[\mathrm{X}_{\mathrm{II}} / \mathrm{Fe}_{\mathrm{I}}\right]$ ratios may be similarly overestimated in our results.

Figure 2 also shows that most $[\mathrm{X} / \mathrm{Fe}]$ ratios have no $T_{\text {eff }}$ dependence. $\mathrm{C}$ and $\mathrm{N}$ may be expected to exhibit such trends resulting from internal processing. The $[\mathrm{Si}$ I/Fe] ratio, derived from the $\mathrm{Si}$ I $3905 \AA$ line, increases by $\sim 0.3$ dex from the coolest stars to the warmest ones in our sample. This trend is in the 
Table 9

Stellar Abundances III

\begin{tabular}{|c|c|c|c|c|c|c|c|c|c|c|c|c|c|c|c|c|}
\hline \multirow[t]{2}{*}{ Species } & \multirow[t]{2}{*}{$Z$} & \multicolumn{5}{|c|}{ VII-68 } & \multicolumn{5}{|c|}{ VIII-24 } & \multicolumn{5}{|c|}{ VIII-44 } \\
\hline & & $\log \epsilon$ & {$[\mathrm{X} / \mathrm{Fe}]$} & $\sigma_{\mu}$ & $\sigma$ & $N$ & $\log \epsilon$ & {$[\mathrm{X} / \mathrm{Fe}]$} & $\sigma_{\mu}$ & $\sigma$ & $N$ & $\log \epsilon$ & {$[\mathrm{X} / \mathrm{Fe}]$} & $\sigma_{\mu}$ & $\sigma$ & $N$ \\
\hline $\mathrm{C}\left({ }^{12} \mathrm{CH}\right)$ & 6 & 5.44 & -0.27 & 0.09 & 0.12 & 2 & 5.64 & -0.18 & 0.32 & 0.32 & 1 & 5.48 & -0.35 & 0.10 & 0.13 & 2 \\
\hline $\mathrm{N}(\mathrm{CN})$ & 7 & 6.83 & 1.72 & 0.20 & 0.20 & 1 & $<6.9$ & $<1.71$ & $\ldots$ & $\ldots$ & $\ldots$ & 6.75 & 1.53 & 0.22 & 0.22 & 1 \\
\hline Si I & 14 & 5.62 & 0.83 & 0.19 & 0.19 & 1 & 5.57 & 0.67 & 0.22 & 0.22 & 1 & 5.73 & 0.83 & 0.20 & 0.20 & 1 \\
\hline $\mathrm{Sc} \mathrm{I}$ & 21 & $\ldots$ & $\ldots$ & $\ldots$ & $\ldots$ & $\ldots$ & $\ldots$ & $\ldots$ & $\ldots$ & $\ldots$ & $\ldots$ & $\ldots$ & $\ldots$ & $\ldots$ & $\ldots$ & $\ldots$ \\
\hline Sc II & 21 & $\ldots$ & $\ldots$ & $\ldots$ & $\ldots$ & $\ldots$ & $\ldots$ & $\ldots$ & $\ldots$ & $\ldots$ & $\ldots$ & $\ldots$ & $\ldots$ & $\ldots$ & $\ldots$ & $\ldots$ \\
\hline Ti I & 22 & 2.25 & 0.02 & 0.20 & 0.34 & 3 & 2.62 & 0.28 & 0.07 & 0.12 & 3 & 2.44 & 0.10 & 0.20 & 0.29 & 2 \\
\hline Ti II & 22 & 2.74 & 0.51 & 0.16 & 0.23 & 2 & 3.04 & 0.70 & 0.10 & 0.14 & 2 & 2.98 & 0.64 & 0.17 & 0.24 & 2 \\
\hline V II & 23 & 1.71 & 0.50 & 0.06 & 0.12 & 4 & 1.79 & 0.46 & 0.18 & 0.25 & 2 & 1.86 & 0.53 & 0.05 & 0.12 & 5 \\
\hline Cr I & 24 & $\ldots$ & $\ldots$ & $\ldots$ & $\ldots$ & $\ldots$ & $\ldots$ & $\ldots$ & $\ldots$ & $\ldots$ & $\ldots$ & $\ldots$ & $\ldots$ & $\ldots$ & $\ldots$ & $\ldots$ \\
\hline Mn I & 25 & 2.60 & -0.10 & 0.09 & 0.15 & 3 & 2.57 & -0.25 & 0.15 & 0.30 & 4 & 2.53 & -0.29 & 0.07 & 0.14 & 4 \\
\hline $\mathrm{Fe} I$ & 26 & 4.78 & -2.72 & 0.07 & 0.17 & 7 & 4.89 & -2.61 & 0.06 & 0.17 & 8 & 4.89 & -2.61 & 0.04 & 0.12 & 9 \\
\hline Co I & 27 & 2.53 & 0.26 & 0.07 & 0.13 & 3 & 2.50 & 0.12 & 0.17 & 0.23 & 2 & 2.61 & 0.23 & 0.17 & 0.30 & 3 \\
\hline Ni I & 28 & $\ldots$ & $\ldots$ & $\ldots$ & $\ldots$ & $\ldots$ & $\ldots$ & $\ldots$ & $\ldots$ & $\ldots$ & $\ldots$ & 3.59 & -0.02 & 0.30 & 0.30 & 1 \\
\hline$Y_{\text {II }}$ & 39 & -0.51 & 0.00 & 0.08 & 0.12 & 2 & -0.37 & 0.03 & 0.10 & 0.14 & 2 & -0.49 & -0.09 & 0.07 & 0.09 & 2 \\
\hline Zr II & 40 & 0.40 & 0.55 & 0.30 & 0.42 & 2 & 0.39 & 0.42 & 0.14 & 0.19 & 2 & 0.29 & 0.32 & 0.09 & 0.13 & 2 \\
\hline La II & 57 & -1.14 & 0.48 & 0.18 & 0.18 & 1 & -1.28 & 0.23 & 0.17 & 0.25 & 2 & -1.33 & 0.18 & 0.09 & 0.16 & 3 \\
\hline Ce II & 58 & $\ldots$ & $\ldots$ & $\ldots$ & $\ldots$ & $\ldots$ & $\ldots$ & $\ldots$ & $\ldots$ & $\ldots$ & $\ldots$ & $\ldots$ & $\ldots$ & $\ldots$ & $\ldots$ & $\ldots$ \\
\hline $\mathrm{Nd}$ II & 60 & $\ldots$ & $\ldots$ & $\ldots$ & $\ldots$ & $\ldots$ & $\ldots$ & $\ldots$ & $\ldots$ & $\ldots$ & $\ldots$ & $\ldots$ & $\ldots$ & $\ldots$ & $\ldots$ & $\ldots$ \\
\hline Eu II & 63 & $<-1.2$ & $<1.03$ & $\ldots$ & $\ldots$ & $\ldots$ & -1.44 & 0.65 & 0.32 & 0.32 & 1 & -1.70 & 0.39 & 0.39 & 0.39 & 1 \\
\hline Ho II & 67 & -1.55 & 0.69 & 0.21 & 0.21 & 1 & $\ldots$ & $\ldots$ & $\ldots$ & $\ldots$ & $\ldots$ & $\ldots$ & $\ldots$ & $\ldots$ & $\ldots$ & $\ldots$ \\
\hline Er II & 68 & $\ldots$ & $\ldots$ & $\ldots$ & $\ldots$ & $\ldots$ & $\ldots$ & $\ldots$ & $\ldots$ & $\ldots$ & $\ldots$ & $\ldots$ & $\ldots$ & $\ldots$ & $\ldots$ & $\ldots$ \\
\hline
\end{tabular}

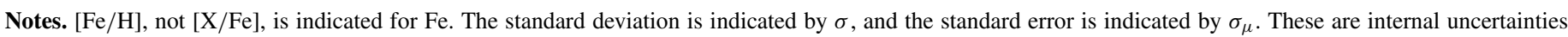
only; see Section 3.3 for a discussion of absolute uncertainties.

Table 10

Stellar Abundances IV

\begin{tabular}{|c|c|c|c|c|c|c|c|c|c|c|c|c|c|c|c|c|}
\hline \multirow[t]{2}{*}{ Species } & \multirow[t]{2}{*}{$Z$} & \multicolumn{5}{|c|}{ IX-49 } & \multicolumn{5}{|c|}{ IX-89 } & \multicolumn{5}{|c|}{$X-3$} \\
\hline & & $\log \epsilon$ & {$[\mathrm{X} / \mathrm{Fe}]$} & $\sigma_{\mu}$ & $\sigma$ & $N$ & $\log \epsilon$ & {$[\mathrm{X} / \mathrm{Fe}]$} & $\sigma_{\mu}$ & $\sigma$ & $N$ & $\log \epsilon$ & {$[\mathrm{X} / \mathrm{Fe}]$} & $\sigma_{\mu}$ & $\sigma$ & $N$ \\
\hline $\mathrm{C}\left({ }^{12} \mathrm{CH}\right)$ & 6 & 5.36 & -0.42 & 0.11 & 0.15 & 2 & 5.62 & -0.26 & 0.18 & 0.25 & 2 & 5.70 & -0.38 & 0.14 & 0.20 & 2 \\
\hline $\mathrm{N}(\mathrm{CN})$ & 7 & 6.87 & 1.69 & 0.24 & 0.24 & 1 & 7.09 & 1.81 & 0.15 & 0.15 & 1 & 7.83 & 2.35 & 0.11 & 0.11 & 1 \\
\hline Si I & 14 & 5.48 & 0.62 & 0.17 & 0.17 & 1 & 5.31 & 0.35 & 0.21 & 0.21 & 1 & 5.95 & 0.79 & 0.14 & 0.14 & 1 \\
\hline Sc I & 21 & $\ldots$ & $\ldots$ & $\ldots$ & $\ldots$ & $\ldots$ & $\ldots$ & $\ldots$ & $\ldots$ & $\ldots$ & $\ldots$ & $\ldots$ & $\ldots$ & $\ldots$ & $\ldots$ & $\ldots$ \\
\hline Sc II & 21 & 1.09 & 0.59 & 0.39 & 0.39 & 1 & $\ldots$ & $\ldots$ & $\ldots$ & $\ldots$ & $\ldots$ & $\ldots$ & $\ldots$ & $\ldots$ & $\ldots$ & $\ldots$ \\
\hline Ti I & 22 & 2.18 & -0.12 & 0.15 & 0.30 & 4 & 2.32 & -0.08 & 0.12 & 0.21 & 3 & 2.68 & 0.08 & 0.09 & 0.16 & 3 \\
\hline Ti II & 22 & 3.07 & 0.77 & 0.09 & 0.13 & 2 & 2.89 & 0.49 & 0.23 & 0.32 & 2 & 3.23 & 0.63 & 0.18 & 0.25 & 2 \\
\hline V II & 23 & 1.72 & 0.44 & 0.04 & 0.09 & 5 & 1.72 & 0.34 & 0.11 & 0.21 & 4 & 1.88 & 0.30 & 0.07 & 0.12 & 3 \\
\hline Cr I & 24 & $\ldots$ & $\ldots$ & $\ldots$ & $\ldots$ & $\ldots$ & $\ldots$ & $\ldots$ & $\ldots$ & $\ldots$ & $\ldots$ & $\ldots$ & $\ldots$ & $\ldots$ & $\ldots$ & $\ldots$ \\
\hline Mn I & 25 & 2.52 & -0.27 & 0.04 & 0.10 & 5 & 2.45 & -0.43 & 0.08 & 0.15 & 4 & 2.68 & -0.40 & 0.10 & 0.23 & 5 \\
\hline $\mathrm{Fe}_{\mathrm{I}}$ & 26 & 4.85 & -2.65 & 0.03 & 0.10 & 8 & 4.95 & -2.55 & 0.09 & 0.25 & 8 & 5.15 & -2.35 & 0.07 & 0.19 & 8 \\
\hline Co I & 27 & 2.32 & -0.02 & 0.15 & 0.26 & 3 & 2.46 & 0.03 & 0.21 & 0.36 & 3 & 2.75 & 0.11 & 0.14 & 0.19 & 2 \\
\hline Ni I & 28 & $\ldots$ & $\ldots$ & $\ldots$ & $\ldots$ & $\ldots$ & $\ldots$ & $\ldots$ & $\ldots$ & $\ldots$ & $\ldots$ & $\ldots$ & $\ldots$ & $\ldots$ & $\ldots$ & $\ldots$ \\
\hline Y II & 39 & -0.57 & -0.13 & 0.08 & 0.11 & 2 & -0.50 & -0.16 & 0.11 & 0.15 & 2 & -0.24 & -0.10 & 0.09 & 0.12 & 2 \\
\hline Zr II & 40 & 0.40 & 0.47 & 0.08 & 0.14 & 3 & 0.35 & 0.33 & 0.14 & 0.19 & 2 & 0.55 & 0.32 & 0.13 & 0.18 & 2 \\
\hline La II & 57 & -1.16 & 0.39 & 0.12 & 0.16 & 2 & -1.31 & 0.14 & 0.12 & 0.17 & 2 & -1.23 & 0.02 & 0.15 & 0.21 & 2 \\
\hline Ce II & 58 & $\ldots$ & $\ldots$ & $\ldots$ & $\ldots$ & $\ldots$ & $\ldots$ & $\ldots$ & $\ldots$ & $\ldots$ & $\ldots$ & $\ldots$ & $\ldots$ & $\ldots$ & $\ldots$ & $\ldots$ \\
\hline $\mathrm{Nd}$ II & 60 & $\ldots$ & $\ldots$ & $\ldots$ & $\ldots$ & $\ldots$ & $\ldots$ & $\ldots$ & $\ldots$ & $\ldots$ & $\ldots$ & $\ldots$ & $\ldots$ & $\ldots$ & $\ldots$ & $\ldots$ \\
\hline Eu II & 63 & -1.29 & 0.84 & 0.40 & 0.40 & 1 & -1.39 & 0.64 & 0.23 & 0.23 & 1 & -1.20 & 0.63 & 0.43 & 0.43 & 1 \\
\hline Ho II & 67 & $\ldots$ & $\ldots$ & $\ldots$ & $\ldots$ & $\ldots$ & -1.41 & 0.66 & 0.38 & 0.38 & 1 & $\ldots$ & $\ldots$ & $\ldots$ & $\ldots$ & $\ldots$ \\
\hline Er II & 68 & $\ldots$ & $\ldots$ & $\ldots$ & $\ldots$ & $\ldots$ & $\ldots$ & $\ldots$ & $\ldots$ & $\ldots$ & $\ldots$ & $\ldots$ & $\ldots$ & $\ldots$ & $\ldots$ & $\ldots$ \\
\hline
\end{tabular}

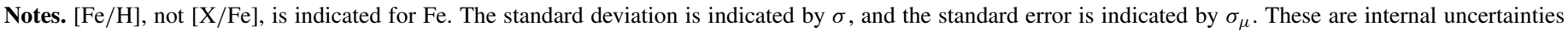
only; see Section 3.3 for a discussion of absolute uncertainties.

opposite sense of what has been found by previous investigators (Cohen et al. 2004; Preston et al. 2006; Lai et al. 2008; Bonifacio et al. 2009; Roederer et al. 2010b). The Si I $3905 \AA$ line is very strong and blended, particularly with $\mathrm{CH}$. Our tests indicate that modeling these blends with a one-dimensional LTE approach could account for the trend in $\mathrm{Si}$ (i.e., reasonable variations in the $\mathrm{CH}$ abundance can account for the deficiency of $\mathrm{Si}$ in the coolest stars), so we discard the Si abundances from further consideration. No heavier species exhibits a correlation with $T_{\text {eff }}$ in $[\mathrm{X} / \mathrm{Fe}]$, except perhaps [Y II/Fe]. This apparent correlation rests strongly on the warmest star, XII-18. Other $[\mathrm{X} / \mathrm{Fe}]$ ratios in this star also appear to be higher than their respective means for 
Table 11

Stellar Abundances V

\begin{tabular}{|c|c|c|c|c|c|c|c|c|c|c|c|c|c|c|c|c|}
\hline \multirow[t]{2}{*}{ Species } & \multirow[t]{2}{*}{$Z$} & \multicolumn{5}{|c|}{ XI-10 } & \multicolumn{5}{|c|}{ XI-19 } & \multicolumn{5}{|c|}{ XI-80 } \\
\hline & & $\log \epsilon$ & {$[\mathrm{X} / \mathrm{Fe}]$} & $\sigma_{\mu}$ & $\sigma$ & $N$ & $\log \epsilon$ & {$[\mathrm{X} / \mathrm{Fe}]$} & $\sigma_{\mu}$ & $\sigma$ & $N$ & $\log \epsilon$ & {$[\mathrm{X} / \mathrm{Fe}]$} & $\sigma_{\mu}$ & $\sigma$ & $N$ \\
\hline $\mathrm{C}\left({ }^{12} \mathrm{CH}\right)$ & 6 & 5.76 & -0.15 & 0.07 & 0.10 & 2 & 5.18 & -0.51 & 0.06 & 0.09 & 2 & 5.13 & -0.58 & 0.06 & 0.08 & 2 \\
\hline $\mathrm{N}(\mathrm{CN})$ & 7 & 6.64 & 1.33 & 0.38 & 0.38 & 1 & 6.56 & 1.46 & 0.13 & 0.13 & 1 & 6.61 & 1.50 & 0.11 & 0.11 & 1 \\
\hline Si I & 14 & 5.75 & 0.76 & 0.14 & 0.14 & 1 & 5.41 & 0.63 & 0.14 & 0.14 & 1 & 5.31 & 0.52 & 0.11 & 0.11 & 1 \\
\hline $\mathrm{Sc} \mathrm{I}$ & 21 & $\ldots$ & $\ldots$ & $\ldots$ & $\ldots$ & $\ldots$ & 0.46 & 0.04 & 0.18 & 0.18 & 1 & 0.63 & 0.20 & 0.16 & 0.16 & 1 \\
\hline Sc II & 21 & $\ldots$ & $\ldots$ & $\ldots$ & $\ldots$ & $\ldots$ & 1.13 & 0.71 & 0.28 & 0.28 & 1 & 0.95 & 0.52 & 0.26 & 0.26 & 1 \\
\hline Ti I & 22 & 2.37 & -0.06 & 0.07 & 0.15 & 4 & 2.38 & 0.17 & 0.07 & 0.14 & 4 & 2.46 & 0.23 & 0.05 & 0.10 & 4 \\
\hline Ti II & 22 & 3.10 & 0.67 & 0.08 & 0.12 & 2 & 2.90 & 0.69 & 0.05 & 0.07 & 2 & 2.89 & 0.66 & 0.06 & 0.09 & 2 \\
\hline V II & 23 & 1.76 & 0.35 & 0.07 & 0.15 & 4 & 1.75 & 0.55 & 0.03 & 0.07 & 5 & 1.67 & 0.46 & 0.03 & 0.06 & 5 \\
\hline Cr I & 24 & $\ldots$ & $\ldots$ & $\ldots$ & $\ldots$ & $\ldots$ & 2.69 & -0.22 & 0.20 & 0.20 & 1 & 2.54 & -0.38 & 0.15 & 0.15 & 1 \\
\hline Mn I & 25 & 2.54 & -0.38 & 0.07 & 0.14 & 4 & 2.50 & -0.19 & 0.04 & 0.09 & 5 & 2.43 & -0.28 & 0.04 & 0.09 & 5 \\
\hline $\mathrm{Fe} \mathrm{I}$ & 26 & 4.98 & -2.52 & 0.05 & 0.15 & 8 & 4.77 & -2.73 & 0.05 & 0.14 & 9 & 4.78 & -2.72 & 0.04 & 0.12 & 9 \\
\hline Co I & 27 & 2.46 & -0.01 & 0.10 & 0.14 & 2 & 2.41 & 0.15 & 0.05 & 0.08 & 3 & 2.29 & 0.02 & 0.04 & 0.08 & 3 \\
\hline Ni I & 28 & $\ldots$ & $\ldots$ & $\ldots$ & $\ldots$ & $\ldots$ & 3.40 & -0.09 & 0.15 & 0.15 & 1 & 3.25 & -0.25 & 0.17 & 0.17 & 1 \\
\hline Y II & 39 & -0.54 & -0.23 & 0.08 & 0.12 & 2 & -0.71 & -0.19 & 0.07 & 0.10 & 2 & -0.79 & -0.28 & 0.06 & 0.08 & 2 \\
\hline Zr II & 40 & 0.31 & 0.24 & 0.21 & 0.29 & 2 & 0.44 & 0.60 & 0.08 & 0.13 & 3 & 0.36 & 0.50 & 0.06 & 0.11 & 3 \\
\hline La II & 57 & -1.37 & 0.04 & 0.14 & 0.20 & 2 & -1.53 & 0.10 & 0.11 & 0.21 & 4 & -1.51 & 0.11 & 0.11 & 0.23 & 4 \\
\hline Ce II & 58 & $\ldots$ & $\ldots$ & $\ldots$ & $\ldots$ & $\ldots$ & -0.69 & 0.46 & 0.14 & 0.14 & 1 & -0.73 & 0.41 & 0.23 & 0.23 & 1 \\
\hline Nd II & 60 & $\ldots$ & $\ldots$ & $\ldots$ & $\ldots$ & $\ldots$ & -0.93 & 0.38 & 0.13 & 0.18 & 2 & -1.21 & 0.09 & 0.32 & 0.32 & 1 \\
\hline Eu II & 63 & -1.75 & 0.25 & 0.33 & 0.33 & 1 & -2.05 & 0.16 & 0.20 & 0.20 & 1 & -2.17 & 0.03 & 0.35 & 0.35 & 1 \\
\hline Ho II & 67 & $\ldots$ & $\ldots$ & $\ldots$ & $\ldots$ & $\ldots$ & -2.02 & 0.23 & 0.19 & 0.19 & 1 & -1.86 & 0.38 & 0.24 & 0.24 & 1 \\
\hline Er II & 68 & $\ldots$ & $\ldots$ & $\ldots$ & $\ldots$ & $\ldots$ & -1.05 & 0.76 & 0.15 & 0.15 & 1 & -0.98 & 0.82 & 0.17 & 0.17 & 1 \\
\hline
\end{tabular}

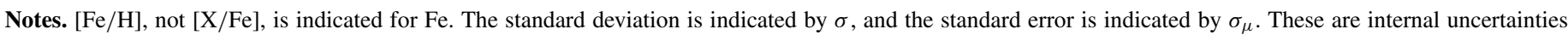
only; see Section 3.3 for a discussion of absolute uncertainties.

Table 12

Stellar Abundances VI

\begin{tabular}{|c|c|c|c|c|c|c|c|c|c|c|c|c|c|c|c|c|}
\hline \multirow[t]{2}{*}{ Species } & \multirow[t]{2}{*}{$Z$} & \multicolumn{5}{|c|}{ XII-8 } & \multicolumn{5}{|c|}{ XII-18 } & \multicolumn{5}{|c|}{ XII-34 } \\
\hline & & $\log \epsilon$ & {$[\mathrm{X} / \mathrm{Fe}]$} & $\sigma_{\mu}$ & $\sigma$ & $N$ & $\log \epsilon$ & {$[\mathrm{X} / \mathrm{Fe}]$} & $\sigma_{\mu}$ & $\sigma$ & $N$ & $\log \epsilon$ & {$[\mathrm{X} / \mathrm{Fe}]$} & $\sigma_{\mu}$ & $\sigma$ & $N$ \\
\hline $\mathrm{C}\left({ }^{12} \mathrm{CH}\right)$ & 6 & 5.08 & -0.46 & 0.04 & 0.04 & 2 & $<6.3$ & $<0.58$ & $\ldots$ & $\ldots$ & $\ldots$ & 5.24 & -0.50 & 0.09 & 0.13 & 2 \\
\hline $\mathrm{N}(\mathrm{CN})$ & 7 & 5.65 & 0.71 & 0.10 & 0.10 & 1 & $<7.9$ & $<2.78$ & $\ldots$ & $\ldots$ & $\ldots$ & 6.44 & 1.30 & 0.24 & 0.24 & 1 \\
\hline Si I & 14 & 5.05 & 0.43 & 0.10 & 0.10 & 1 & 5.63 & 0.80 & 0.21 & 0.21 & 1 & 5.40 & 0.58 & 0.14 & 0.14 & 1 \\
\hline Sc I & 21 & 0.40 & 0.14 & 0.10 & 0.10 & 1 & $\ldots$ & $\ldots$ & $\ldots$ & $\ldots$ & $\ldots$ & $\ldots$ & $\ldots$ & $\ldots$ & $\ldots$ & $\ldots$ \\
\hline Sc II & 21 & 0.80 & 0.54 & 0.10 & 0.10 & 1 & $\ldots$ & $\ldots$ & $\ldots$ & $\ldots$ & $\ldots$ & 1.03 & 0.57 & 0.19 & 0.19 & 1 \\
\hline Ti I & 22 & 2.22 & 0.16 & 0.14 & 0.27 & 4 & 2.50 & 0.23 & 0.15 & 0.25 & 3 & 2.38 & 0.12 & 0.11 & 0.23 & 4 \\
\hline Ti II & 22 & 2.93 & 0.87 & 0.07 & 0.10 & 2 & 2.99 & 0.71 & 0.18 & 0.25 & 2 & 2.94 & 0.68 & 0.07 & 0.09 & 2 \\
\hline V II & 23 & 1.64 & 0.60 & 0.10 & 0.23 & 5 & 1.97 & 0.71 & 0.05 & 0.11 & 5 & 1.78 & 0.54 & 0.06 & 0.12 & 5 \\
\hline CrI & 24 & 2.20 & -0.55 & 0.10 & 0.10 & 1 & $\ldots$ & $\ldots$ & $\ldots$ & $\ldots$ & $\ldots$ & $\ldots$ & $\ldots$ & $\ldots$ & $\ldots$ & $\ldots$ \\
\hline Mn I & 25 & 2.15 & -0.39 & 0.04 & 0.10 & 5 & 2.39 & -0.37 & 0.13 & 0.25 & 4 & 2.50 & -0.24 & 0.07 & 0.15 & 5 \\
\hline $\mathrm{Fe}_{\mathrm{I}}$ & 26 & 4.61 & -2.89 & 0.06 & 0.18 & 9 & 4.82 & -2.68 & 0.04 & 0.09 & 5 & 4.81 & -2.69 & 0.05 & 0.15 & 9 \\
\hline Co I & 27 & 2.21 & 0.11 & 0.23 & 0.39 & 3 & 2.81 & 0.50 & 0.17 & 0.23 & 2 & 2.55 & 0.25 & 0.06 & 0.10 & 3 \\
\hline Ni I & 28 & 3.25 & -0.08 & 0.10 & 0.10 & 1 & $\ldots$ & $\ldots$ & $\ldots$ & $\ldots$ & $\ldots$ & 3.31 & -0.22 & 0.21 & 0.21 & 1 \\
\hline$Y_{\text {II }}$ & 39 & -0.80 & -0.12 & 0.20 & 0.28 & 2 & -0.44 & 0.03 & 0.08 & 0.12 & 2 & -0.47 & 0.01 & 0.09 & 0.13 & 2 \\
\hline Zr II & 40 & 0.27 & 0.58 & 0.17 & 0.29 & 3 & 0.59 & 0.69 & 0.27 & 0.27 & 1 & 0.43 & 0.54 & 0.11 & 0.20 & 3 \\
\hline La II & 57 & -1.38 & 0.41 & 0.03 & 0.06 & 4 & -1.08 & 0.50 & 0.21 & 0.21 & 1 & -1.34 & 0.25 & 0.17 & 0.33 & 4 \\
\hline Ce II & 58 & -0.85 & 0.46 & 0.10 & 0.10 & 1 & $\ldots$ & $\ldots$ & $\ldots$ & $\ldots$ & $\ldots$ & $\ldots$ & $\ldots$ & $\ldots$ & $\ldots$ & $\ldots$ \\
\hline $\mathrm{Nd}$ II & 60 & -0.95 & 0.52 & 0.10 & 0.14 & 2 & $\ldots$ & $\ldots$ & $\ldots$ & $\ldots$ & $\ldots$ & -1.12 & 0.15 & 0.30 & 0.30 & 1 \\
\hline Eu II & 63 & -1.75 & 0.62 & 0.10 & 0.10 & 1 & -1.70 & 0.46 & 0.36 & 0.36 & 1 & -1.93 & 0.24 & 0.41 & 0.41 & 1 \\
\hline Ho II & 67 & -1.85 & 0.56 & 0.10 & 0.10 & 1 & -1.37 & 0.83 & 0.37 & 0.37 & 1 & -1.94 & 0.27 & 0.36 & 0.36 & 1 \\
\hline Er II & 68 & -1.30 & 0.67 & 0.10 & 0.10 & 1 & $\ldots$ & $\ldots$ & $\ldots$ & $\ldots$ & $\ldots$ & -1.16 & 0.61 & 0.36 & 0.36 & 1 \\
\hline
\end{tabular}

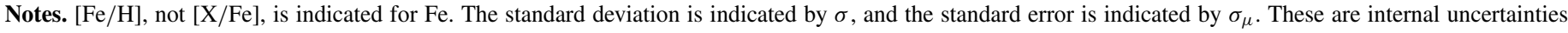
only; see Section 3.3 for a discussion of absolute uncertainties.

M92 and the $[\mathrm{Fe} / \mathrm{H}]$ ratio seems to be lower than the mean trend defined by the other 18 stars, so we dismiss this trend as well.

Following recommendations by Cayrel et al. (2004) and Roederer et al. (2010b), we artificially increase the Mn I abundances derived from the 4030, 4033, and $4034 \AA$ lines by 0.3 dex to bring them in better agreement with abundances derived from other Mn I indicators. This offset is reflected in all tables and figures.

\subsection{Comparison of Individual Stars with Previous Studies}

VII-18 has been studied in detail by Shetrone et al. (2001) and Johnson (2002). Both of these studies derived $[\mathrm{Fe} \mathrm{I} / \mathrm{H}]$ higher by $\approx 0.5$ dex. The neutral to neutral metal ratios (i.e., $\left[\mathrm{X}_{\mathrm{I}} / \mathrm{Fe}_{\mathrm{I}}\right]$ ) are generally in agreement to better than 0.2 dex. Correcting for differences in the $\log (g f)$ values, we find that the singly ionized to singly ionized ratios among the heavy $n$-capture elements are 
Table 13

Stellar Abundances VII

\begin{tabular}{|c|c|c|c|c|c|c|}
\hline \multirow[t]{2}{*}{ Species } & \multirow[t]{2}{*}{$Z$} & \multicolumn{5}{|c|}{ Bu166 } \\
\hline & & $\log \epsilon$ & {$[\mathrm{X} / \mathrm{Fe}]$} & $\sigma_{\mu}$ & $\sigma$ & $N$ \\
\hline $\mathrm{C}\left({ }^{12} \mathrm{CH}\right)$ & 6 & 5.73 & -0.14 & 0.08 & 0.11 & 2 \\
\hline $\mathrm{N}(\mathrm{CN})$ & 7 & $<6.5$ & $<1.26$ & $\ldots$ & $\ldots$ & \\
\hline Si I & 14 & 5.64 & 0.69 & 0.16 & 0.16 & 1 \\
\hline Sc I & 21 & $\ldots$ & $\ldots$ & $\ldots$ & $\ldots$ & \\
\hline Sc II & 21 & $\ldots$ & $\ldots$ & $\ldots$ & $\ldots$ & $\ldots$ \\
\hline Ti I & 22 & 2.38 & -0.01 & 0.09 & 0.18 & 4 \\
\hline Ti II & 22 & 2.88 & 0.49 & 0.09 & 0.12 & 2 \\
\hline V II & 23 & 1.92 & 0.55 & 0.06 & 0.12 & 4 \\
\hline Cr I & 24 & $\ldots$ & $\ldots$ & $\ldots$ & $\ldots$ & $\ldots$ \\
\hline Mn I & 25 & 2.49 & -0.38 & 0.15 & 0.25 & 3 \\
\hline $\mathrm{Fe} \mathrm{I}$ & 26 & 4.94 & -2.56 & 0.08 & 0.19 & 6 \\
\hline Co I & 27 & 2.60 & 0.17 & 0.09 & 0.16 & 3 \\
\hline Ni I & 28 & $\ldots$ & $\ldots$ & $\ldots$ & $\ldots$ & $\ldots$ \\
\hline Y II & 39 & -0.58 & -0.23 & 0.17 & 0.24 & 2 \\
\hline Zr II & 40 & 0.16 & 0.14 & 0.15 & 0.21 & 2 \\
\hline La II & 57 & -1.26 & 0.20 & 0.17 & 0.24 & 2 \\
\hline Ce II & 58 & $\ldots$ & $\ldots$ & $\ldots$ & $\ldots$ & $\ldots$ \\
\hline Nd II & 60 & $\ldots$ & $\ldots$ & $\ldots$ & $\ldots$ & $\ldots$ \\
\hline Eu II & 63 & $<-1.5$ & $<0.57$ & $\ldots$ & $\ldots$ & \\
\hline Ho II & 67 & $\ldots$ & $\ldots$ & $\ldots$ & $\ldots$ & $\ldots$ \\
\hline Er II & 68 & $\ldots$ & $\ldots$ & $\ldots$ & $\ldots$ & $\ldots$ \\
\hline
\end{tabular}

Notes. $[\mathrm{Fe} / \mathrm{H}]$, not $[\mathrm{X} / \mathrm{Fe}]$, is indicated for $\mathrm{Fe}$. The standard deviation is indicated by $\sigma$, and the standard error is indicated by $\sigma_{\mu}$. These are internal uncertainties only; see Section 3.3 for a discussion of absolute uncertainties.

Table 14

Impact of Model Atmosphere Uncertainties on Abundances

\begin{tabular}{lcccc}
\hline \hline Species & $\begin{array}{c}\Delta T= \\
\pm 100 \mathrm{~K}\end{array}$ & $\begin{array}{c}\Delta \log g= \\
\pm 0.3\end{array}$ & $\begin{array}{c}\Delta v_{t}= \\
\pm 0.3 \mathrm{~km} \mathrm{~s}^{-1}\end{array}$ & $\begin{array}{c}\Delta[\mathrm{M} / \mathrm{H}]= \\
\pm 0.4 \mathrm{dex}\end{array}$ \\
\hline $\log \epsilon$ (Ti II) & \pm 0.02 & \pm 0.09 & $\mp 0.15$ & \pm 0.01 \\
$\log \epsilon$ (V II $)$ & \pm 0.02 & \pm 0.13 & $\mp 0.05$ & \pm 0.05 \\
$\log \epsilon$ (Fe I $)$ & \pm 0.10 & \pm 0.01 & $\mp 0.03$ & \pm 0.05 \\
$\log \epsilon$ (La II $)$ & \pm 0.04 & \pm 0.09 & $\mp 0.01$ & \pm 0.05 \\
$\log \epsilon$ (Eu II $)$ & \pm 0.03 & \pm 0.05 & $\mp 0.01$ & \pm 0.05 \\
\hline
\end{tabular}

also in agreement within 0.2 dex. The $\left[\mathrm{X}_{\mathrm{II}} / \mathrm{Fe} \mathrm{I}\right]$ ratios are higher by $0.4-0.6 \mathrm{dex}$ in Shetrone et al. and higher by $0.2-0.4 \mathrm{dex}$ in Johnson. This leads us to suspect that our [X II $\left./ \mathrm{Fe}_{\mathrm{I}}\right]$ ratios may be overestimated by $\sim 0.4$ dex in VII- 18 . Since this is the coolest star in our sample, we do not apply a universal offset based on these comparisons to all of our [X II/Fe I] ratios, but we caution that the absolute values of these ratios are likely overestimated.

Langer et al. (1998) presented clear evidence for a $0.18 \pm$ 0.01 dex $(\sigma=0.12)$ overabundance in Ca I, Sc II, Ti I, Ti II, Cr I, Fe I, Fe II, Fe II, Co I, and Ni I in XI-19 relative to XII-8 and V-45 in M92. These three stars have very similar $B-V$ colors and $V$ magnitudes. XI-19 and XII- 8 are also included in our study. Examining the line-by-line differential abundances for Sc I to Ni I (31 lines) in these two stars, we find a mean difference of $0.17 \pm 0.03$ dex $(\sigma=0.16)$, identical to that found by Langer et al. Since there is a clear systematic trend of Fe abundance with $T_{\text {eff }}$ in our sample, our offset should not be taken too literally. After removing the trend there is still a residual dispersion of $0.11 \mathrm{dex}$ in $\mathrm{Fe}$ at a given $T_{\text {eff }}$. We should not expect to probe star-to-star dispersion at a level smaller than this, which renders the offset between XI-19 and XII-8 only mildly significant. The offset discovered by Langer et al. is still unexplained at present.
Table 15

M92 Mean Abundance Ratios

\begin{tabular}{|c|c|c|c|c|}
\hline Ratio & Mean & $\sigma_{\mu}$ & $\sigma$ & $N$ \\
\hline$[\mathrm{C} / \mathrm{Fe}]$ & -0.40 & 0.04 & 0.16 & 18 \\
\hline$[\mathrm{N} / \mathrm{Fe}]$ & +1.55 & 0.10 & 0.36 & 14 \\
\hline$[\mathrm{Si} \mathrm{I} / \mathrm{Fe}]$ & +0.63 & 0.04 & 0.16 & 19 \\
\hline$[\mathrm{Sc} \mathrm{I} / \mathrm{Fe}]$ & +0.16 & 0.04 & 0.07 & 4 \\
\hline$[\mathrm{Sc}$ II $/ \mathrm{Fe}]$ & +0.62 & 0.03 & 0.09 & 8 \\
\hline [Ti I/Fe] & +0.08 & 0.03 & 0.11 & 19 \\
\hline [Ti II/Fe] & +0.71 & 0.03 & 0.12 & 19 \\
\hline$[\mathrm{V}$ II $/ \mathrm{Fe}]$ & +0.53 & 0.02 & 0.10 & 19 \\
\hline$[\mathrm{Cr} \mathrm{I} / \mathrm{Fe}]$ & -0.38 & 0.08 & 0.16 & 4 \\
\hline$[\mathrm{Mn} \mathrm{I} / \mathrm{Fe}]$ & -0.28 & 0.04 & 0.16 & 19 \\
\hline$[\mathrm{Fe} \mathrm{I} / \mathrm{H}]^{\mathrm{a}}$ & -2.70 & 0.03 & 0.14 & 19 \\
\hline$[\mathrm{Co} / \mathrm{Fe}]$ & +0.15 & 0.03 & 0.14 & 19 \\
\hline$[\mathrm{Ni}$ I/Fe] & -0.10 & 0.03 & 0.08 & 9 \\
\hline [Y II/Fe] & -0.07 & 0.03 & 0.12 & 19 \\
\hline$[\mathrm{Zr}$ II $/ \mathrm{Fe}]$ & +0.47 & 0.03 & 0.14 & 19 \\
\hline$[\mathrm{La}$ II $/ \mathrm{Fe}]$ & +0.36 & 0.04 & 0.17 & 19 \\
\hline$[\mathrm{Ce}$ II $/ \mathrm{Fe}]$ & +0.46 & 0.04 & 0.07 & 4 \\
\hline$[\mathrm{Nd}$ II $/ \mathrm{Fe}]$ & +0.43 & 0.08 & 0.20 & 6 \\
\hline [Eu II/Fe] & +0.54 & 0.06 & 0.23 & 16 \\
\hline [Ho II/Fe] & +0.56 & 0.09 & 0.28 & 9 \\
\hline$[\mathrm{Er}$ II $/ \mathrm{Fe}]$ & +0.76 & 0.07 & 0.17 & 7 \\
\hline
\end{tabular}

Note. ${ }^{a}$ The absolute value here is tied to the $[\mathrm{Fe} / / \mathrm{H}]$ derived for XII-8. The uncertainties on that quantity are $\sigma_{\mu}=0.06$ and $\sigma=0.18$. See Section 3.3 and the Appendix for details.

\section{RESULTS}

We have derived abundances of up to 21 species of 19 elements in each of 19 stars in M92 from a differential analysis. Our goal is to search for star-to-star dispersion among the $n$-capture abundances. In this section we analyze the degree of homogeneity of our abundance measurements.

\subsection{Homogeneity of the Fe-group Elements}

As discussed in Section 3.4, we find a range of $[\mathrm{Fe} \mathrm{I} / \mathrm{H}]$ ratios in M92; this is an artifact of our analysis and does not reflect a genuine spread in Fe. The other Fe-group elements examined $(\mathrm{Sc}-\mathrm{Ni})$ exhibit similar trends, but the $[\mathrm{X} / \mathrm{Fe}]$ ratios are generally constant across all $T_{\text {eff }}$. The dispersion in each of these ratios, listed in Table 15 , is accordingly very small. For well-determined means (i.e., $[\mathrm{X} / \mathrm{Fe}]$ is measured in $\geqslant 5$ stars), the standard deviation is modest, $0.07 \leqslant \sigma \leqslant 0.16 \mathrm{dex}$, and consistent with observational uncertainty. Our data reveal no star-to-star dispersion among the Fe-group elements, and we regard this range of standard deviations as the smallest level of dispersion that can be probed by our data.

\subsection{Dispersion of the Heavy n-capture Elements}

The elements $\mathrm{Y}$ and $\mathrm{Zr}$ are thought to be mostly produced by $n$-capture nucleosynthesis. Our analysis finds no evidence of dispersion in the $[\mathrm{Y} / \mathrm{Fe}]$ or $[\mathrm{Zr} / \mathrm{Fe}]$ abundance ratios. Their standard deviation, $0.12 \leqslant \sigma \leqslant 0.14 \mathrm{dex}$, is the same as found for the Fe-group elements.

The heavy-elements La-Er paint a more complex picture. Their $[\mathrm{X} / \mathrm{Fe}]$ star-to-star dispersion is larger, $0.17 \leqslant \sigma \leqslant$ $0.28 \mathrm{dex}$, but this may reflect the difficulty in deriving their abundances. Fewer lines are typically available for analysis (only 1 in the case of Ce II, Eu II, Ho II, and Er II), and these lines are often weaker and more blended than the Fe-group element lines that we have analyzed. 

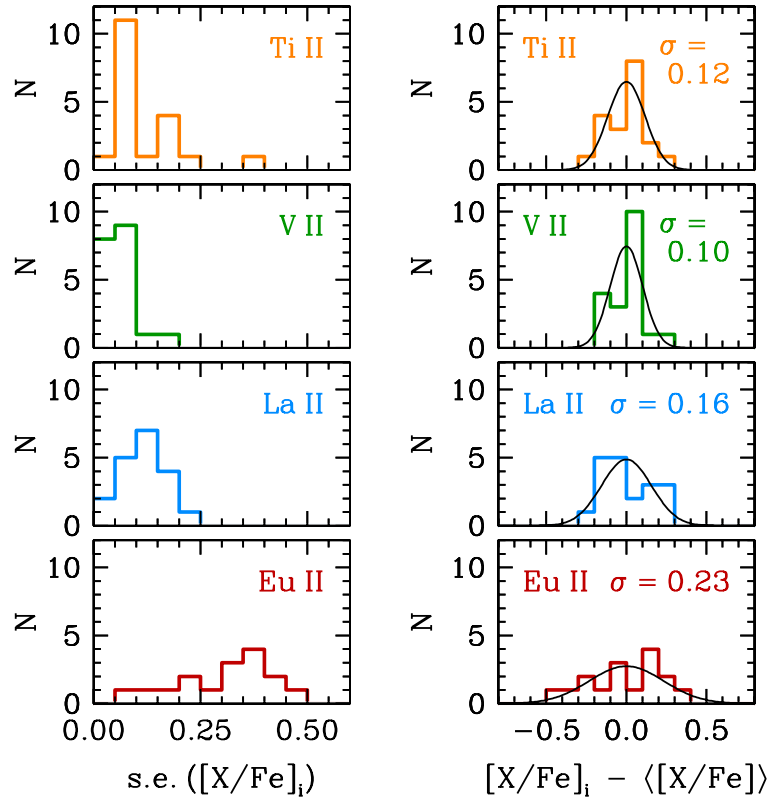

Figure 3. Histograms of the standard error (left) and deviation from the mean $[\mathrm{X} / \mathrm{Fe}]$ abundance ratio (right) for Ti II, V II, La II, and Eu II. The left panels indicate that the median and mode of the (internal) standard error distributions increase from approximately 0.10 dex for Ti II and V II to approximately $0.15 \mathrm{dex}$ for La II and 0.35 dex for Eu II. The (internal) standard deviation, $\sigma$, is shown in the right set of panels along with a Gaussian fit to each distribution. The increase in individual uncertainties can account for much of the broadening of the $[\mathrm{X} / \mathrm{Fe}]$ distributions for La II and Eu II, but it cannot account for correlations between the abundance ratios shown in subsequent figures.

(A color version of this figure is available in the online journal.)

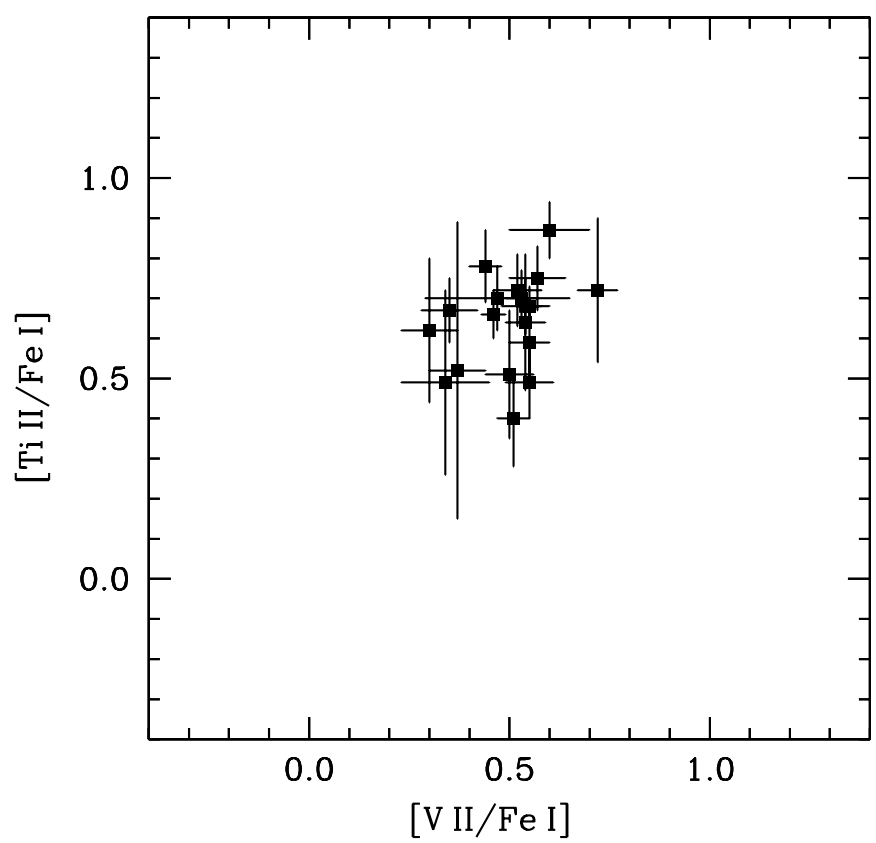

Figure 4. Comparison of the [Ti II/Fe I] and [V II/Fe I] ratios. Only the internal (i.e., star-to-star) uncertainties are shown. There is no significant correlation.

To demonstrate that this may explain at least part of the larger dispersion, in Figure 3 we plot histograms of the standard error on $[\mathrm{X} / \mathrm{Fe}]_{i}$ and the deviation of $[\mathrm{X} / \mathrm{Fe}]_{i}$ from the mean $[\mathrm{X} / \mathrm{Fe}]$. Four ratios are shown: [Ti II $\left./ \mathrm{Fe}_{\mathrm{I}}\right]$, [V II $\left./ \mathrm{Fe}_{\mathrm{I}}\right]$, [La II/Fe I], and [Eu II/Fe I]. The mean, median, and mode of the standard error distributions for $[\mathrm{Ti} / \mathrm{Fe}]$ and $[\mathrm{V} / \mathrm{Fe}]$ are all $\leqslant 0.10 \mathrm{dex}$, and this is reflected in the dispersion about the mean

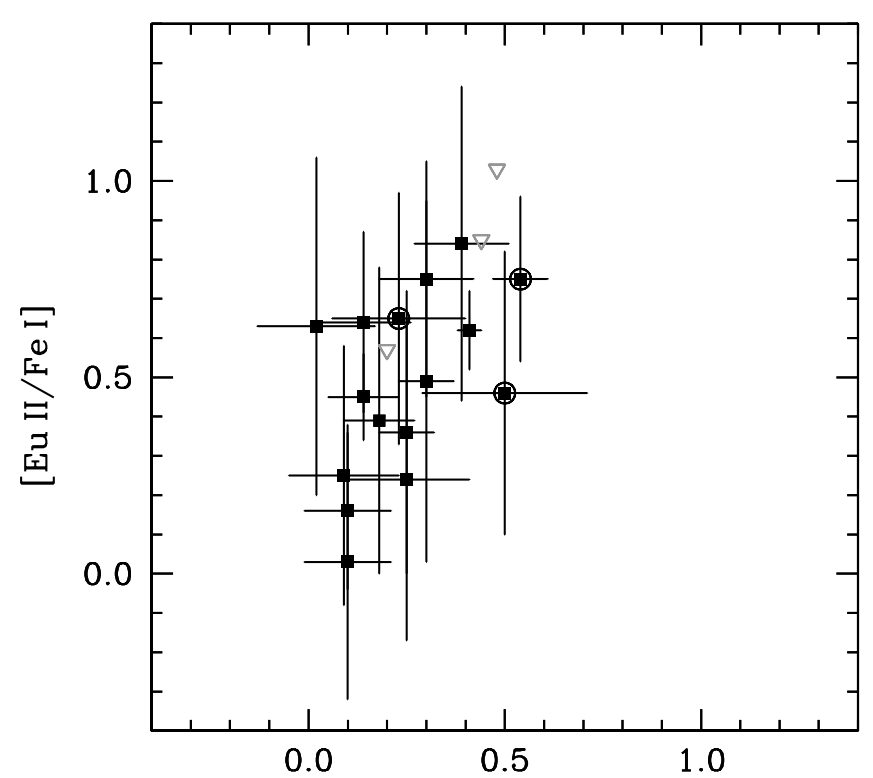

[La II/Fe I]

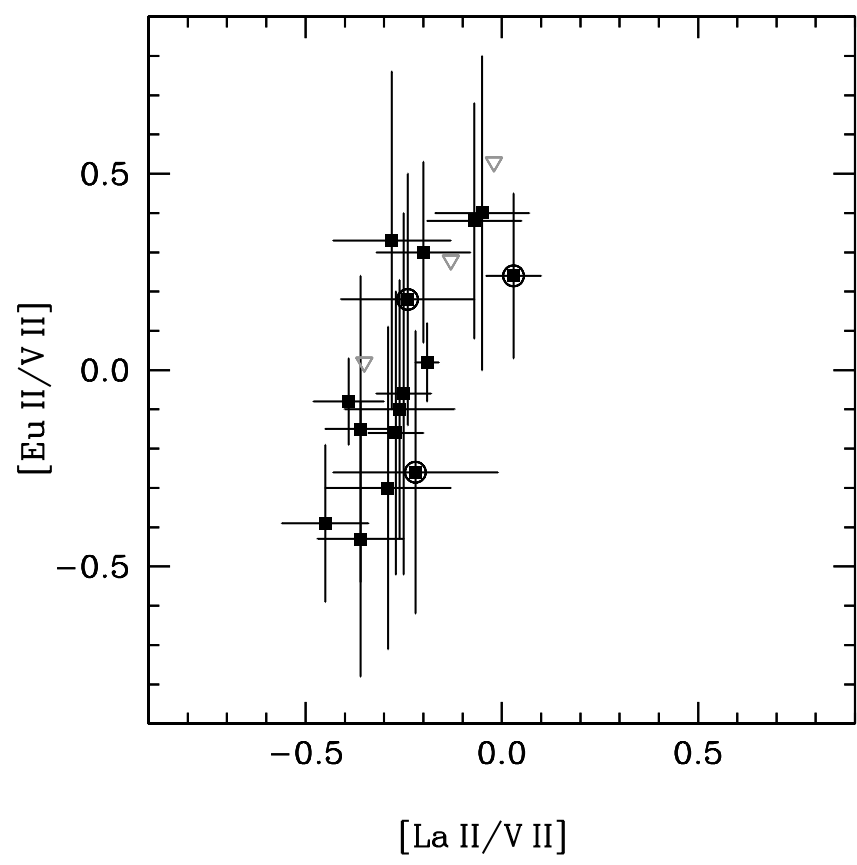

Figure 5. Comparison of the $\left[\mathrm{La}\right.$ II $\left./ \mathrm{Fe}_{\mathrm{I}}\right]$ and $[\mathrm{Eu} \mathrm{II} / \mathrm{Fe} \mathrm{I}]$ ratios (top) and the [La II/V II] and [Eu II/V II] ratios (bottom). Filled squares represent detections and downward-facing open triangles represent upper limits. Open circles indicate probable AGB stars. The correlation is significant whether using a neutral Fe-group abundance indicator or a singly ionized one, indicating that the correlation is unlikely to be an artifact of our abundance analysis. Only the internal (i.e., star-to-star) uncertainties are shown.

$[\mathrm{Ti} / \mathrm{Fe}]$ and $[\mathrm{V} / \mathrm{Fe}]$ ratios, $\sigma=0.12$ and 0.10 dex, respectively. These measures of the standard error distribution are larger for $\mathrm{La}$ and $\mathrm{Eu}, \approx 0.15$ and 0.35 dex, respectively, and this is also reflected in the dispersion about the mean $[\mathrm{La} / \mathrm{Fe}]$ and $[\mathrm{Eu} / \mathrm{Fe}]$ ratios, $\sigma=0.16$ and 0.23 dex, respectively. Thus, it would seem that the larger dispersion observed in $[\mathrm{La} / \mathrm{Fe}]$ and $[\mathrm{Eu} / \mathrm{Fe}]$ can be partially attributed to the larger measurement uncertainties.

If this is the only source of dispersion, we would also expect these ratios to be uncorrelated with one another. Figure 4 demonstrates that this holds true for Ti II and V II. The linear 


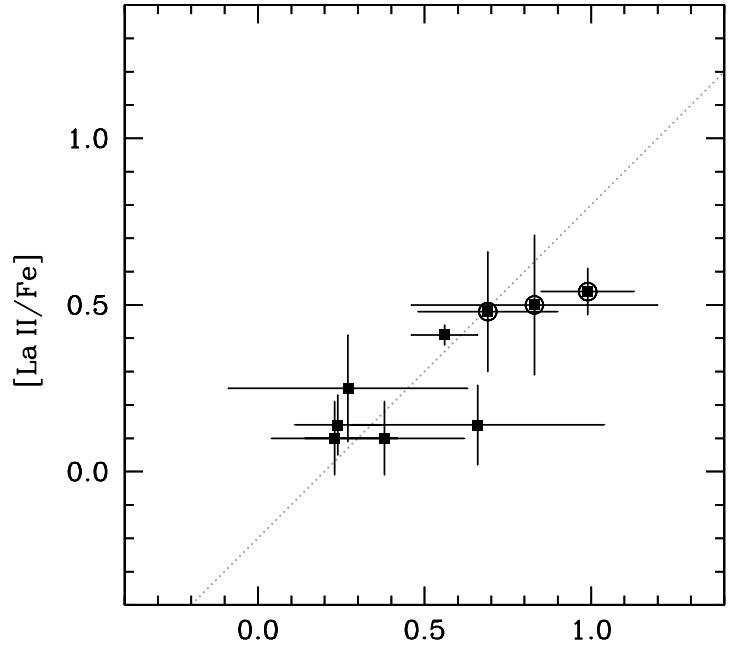

[Ho II $/ \mathrm{Fe}]$

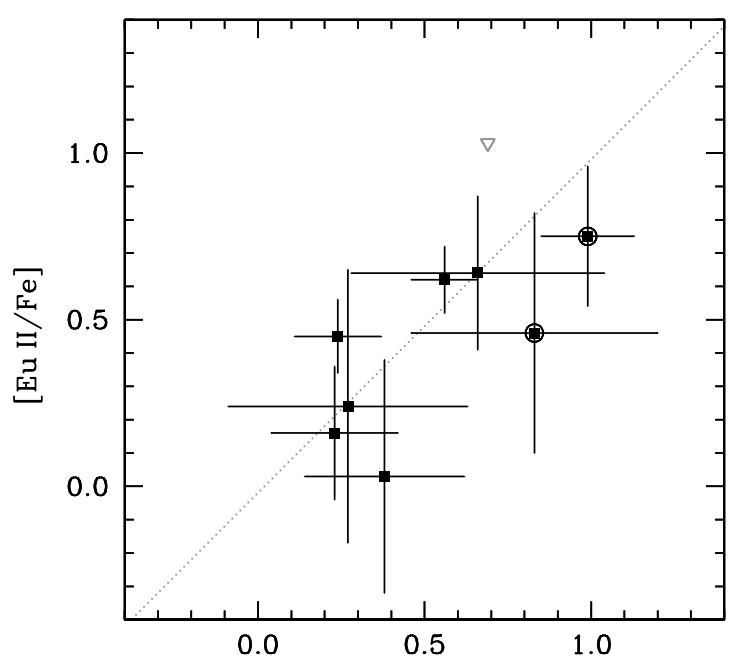

[Ho II $/ \mathrm{Fe}]$

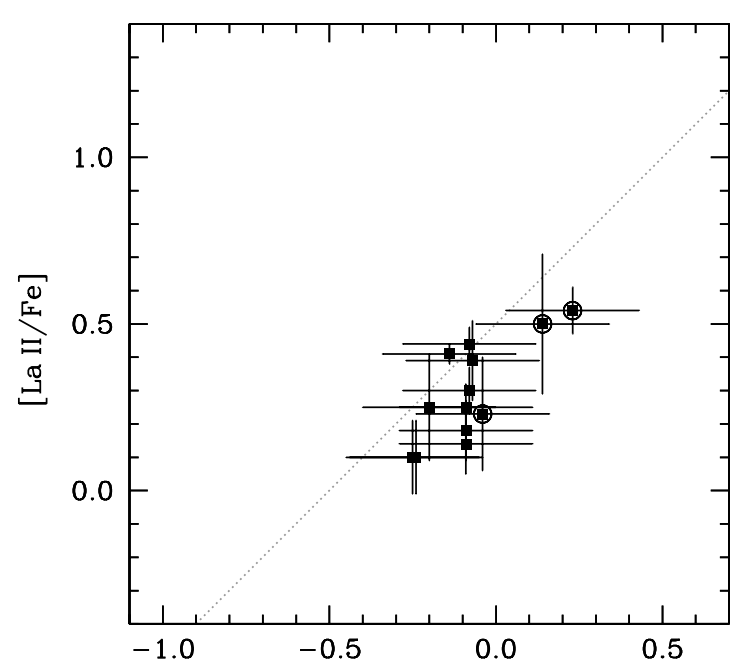

$[\mathrm{Ba} \mathrm{II} / \mathrm{Fe}]$

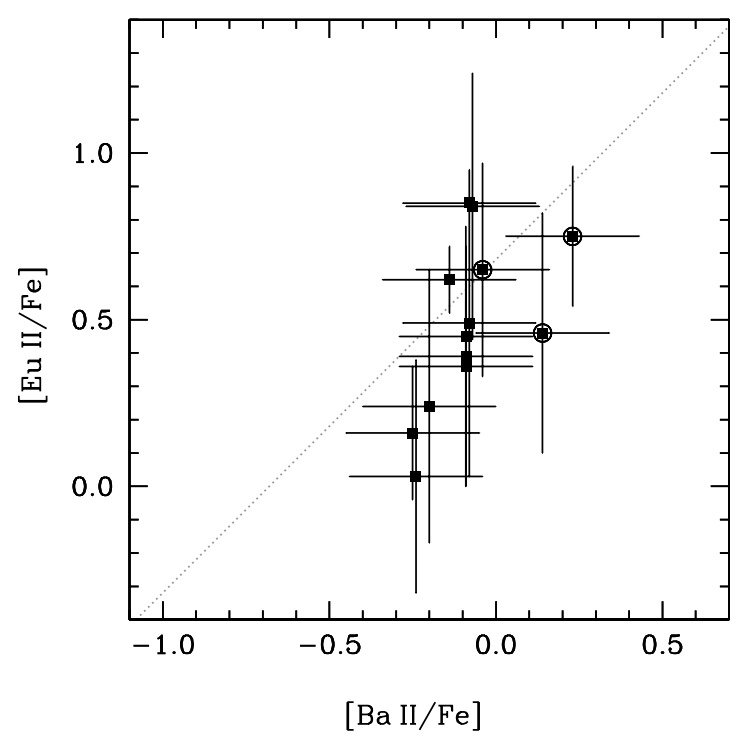

Figure 6. Comparison of the $[\mathrm{La} / \mathrm{Fe}]$ and $[\mathrm{Eu} / \mathrm{Fe}]$ ratios against $[\mathrm{Ho} / \mathrm{Fe}]$ and $[\mathrm{Ba} / \mathrm{Fe}]$. Open circles indicate probable AGB stars. Dotted lines indicate a 1:1 correlation offset by the mean $[\mathrm{Ho} / \mathrm{La}]$ and $[\mathrm{Ho} / \mathrm{Eu}]$ ratios, +0.20 and +0.02 dex, respectively, or the mean $[\mathrm{Ba} / \mathrm{La}]$ and $[\mathrm{Ba} / \mathrm{Eu}]$ ratios, -0.50 and $-0.68 \mathrm{dex}$, respectively. All

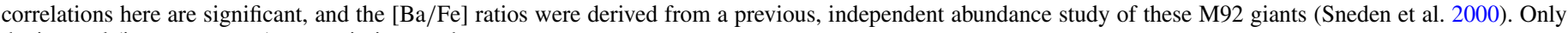
the internal (i.e., star-to-star) uncertainties are shown.

correlation coefficient $r$ (e.g., Bevington \& Robinson 2003) for these variables is 0.31 , indicating a probability $P_{c}$ of $20 \%$ that the $[\mathrm{Ti} \mathrm{II} / \mathrm{Fe} \mathrm{I}]$ and $[\mathrm{V} \mathrm{II} / \mathrm{Fe} \mathrm{I}]$ ratios could have come from an uncorrelated parent population. This correlation is not significant. Figure 5 indicates otherwise for La II and Eu II. [Eu II/Fe I] and [La II/Fe I] correlate with one another, yielding $r=0.49$ and a probability of only $5 \%$ that they were drawn from an uncorrelated parent population. This is perhaps moderately significant.

Figure 6 illustrates that La II and Eu II also correlate strongly with Ho II. Furthermore, La II and Eu II each correlate strongly with the Ba II abundances derived by Sneden et al. (2000), also shown in Figure 6. This is an important point since the Sneden et al. $[\mathrm{Ba} / \mathrm{Fe}]$ ratios were derived from an analysis that obtained the stellar model atmosphere parameters from a different set of photometry (and color- $T_{\text {eff }}$ relations) than we have used. Such a correlation is unlikely to emerge unless it is a genuine feature of the stars being studied. These correlation coefficients are also listed in Table 16.
Figure 7 demonstrates that dispersion in the heavy $n$-capture elements is discernible without a rigorous abundance analysis. Two spectra are shown covering the wavelength regions surrounding several of the lines used in our analysis. These stars, XII-8 and XI-80, have nearly identical $(V-K)$ colors. Their Fe-group elements have similar strengths, indicating that the photometry and $T_{\text {eff }}$ are not in serious error. The lines of Eu II and La II in XII-8 are stronger than those in XI-80, suggesting an intrinsic difference. Note that the Ti II, Y II, and Zr II lines are not significantly different, and the Sc II line shows the opposite effect as La II and Eu II. If one of these stars lies on the RGB and the other on the AGB, we could expect to see consistently different line strengths in all ionized species, which is not the case.

Random uncertainties in our estimation of $T_{\text {eff }}($ or $V-K), \log$ $g, v_{t}$, or $[\mathrm{M} / \mathrm{H}]$ for many stars in the sample could in principle lead to correlated ratios of $[\mathrm{La} / \mathrm{Fe}]$ and $[\mathrm{Eu} / \mathrm{Fe}]$. We can exclude this explanation according to the results of our tests presented in Table 14. To account for a dispersion of $0.3 \mathrm{dex}$ in $[\mathrm{La} / \mathrm{Fe}]$ $(0.4$ dex in $[\mathrm{Eu} / \mathrm{Fe}])$, corresponding to about half the full range 

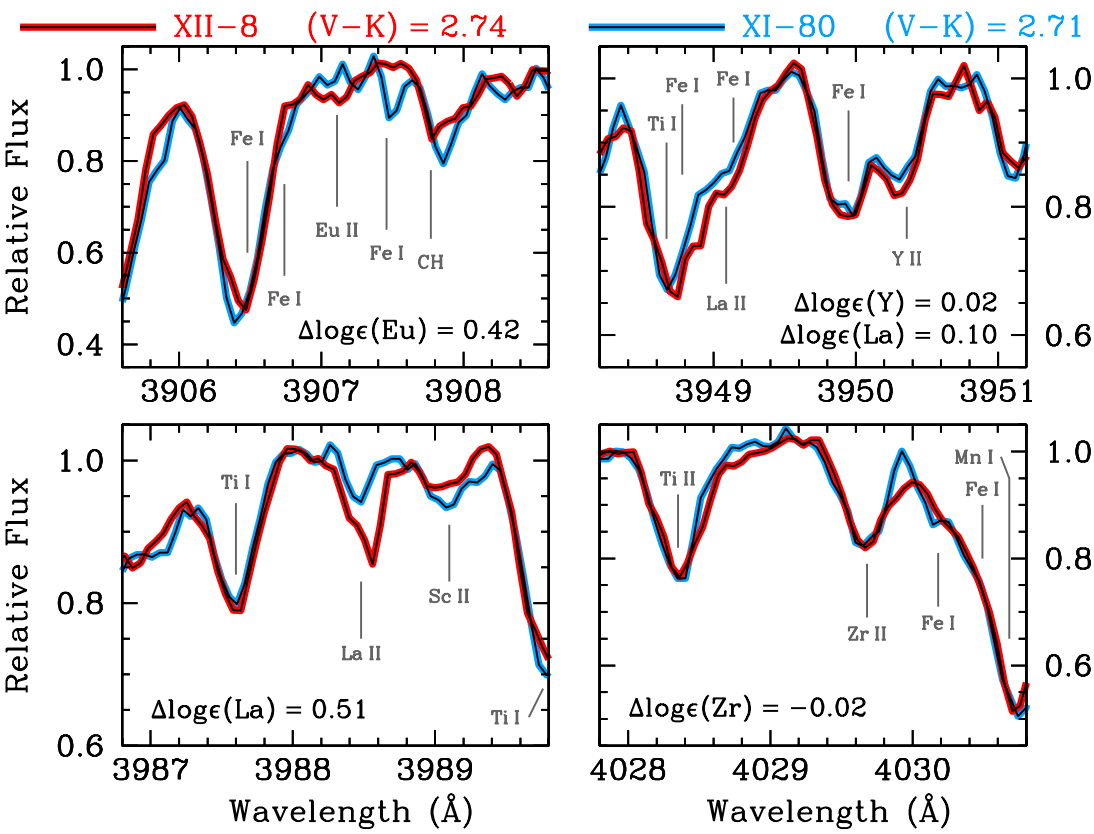

Figure 7. Comparison of the spectra of two stars with contrasting heavy-element abundances. The two stars, XII- 8 and XI-80, have very similar colors and temperatures, yet their Eu and La abundance differentials are large.

(A color version of this figure is available in the online journal.)

Table 16

Correlations Among Abundance Ratios

\begin{tabular}{|c|c|c|c|c|}
\hline \multirow[t]{2}{*}{ Ratio } & \multicolumn{2}{|c|}{ All Stars } & \multicolumn{2}{|c|}{ Excluding Probable AGB Stars } \\
\hline & {$[\mathrm{La} \mathrm{II} / \mathrm{Fe} \mathrm{I}]$} & {$[\mathrm{Eu} \mathrm{II} / \mathrm{Fe} \mathrm{I}]$} & [La II/Fe I $]$ & {$[\mathrm{Eu}$ II $/ \mathrm{Fe} \mathrm{I}]$} \\
\hline$[\mathrm{C} / \mathrm{Fe} \mathrm{I}]$ & $(-0.11,18,0.66)$ & $(0.35,15,0.21)$ & $(-0.24,15,0.39)$ & $(0.32,13,0.28)$ \\
\hline$\left[\mathrm{N} / \mathrm{Fe}_{\mathrm{I}}\right]$ & $(-0.22,14,0.45)$ & $(0.27,12,0.40)$ & $(-0.36,13,0.23)$ & $(0.25,12,0.43)$ \\
\hline$\left[\mathrm{Na} I / \mathrm{Fe}_{\mathrm{I}}\right]^{\mathrm{a}}$ & $(-0.02,13,0.95)$ & $(-0.24,12,0.45)$ & $(-0.13,10,0.71)$ & $(-0.46,9,0.21)$ \\
\hline$[\mathrm{Ba} \text { II } / \mathrm{Fe} \mathrm{I}]^{\mathrm{a}}$ & $(0.78,13,0.0015)$ & $(0.66,12,0.020)$ & $(0.57,10,0.08)$ & $(0.78,9,0.014)$ \\
\hline [La II/Fe I] & $\ldots$ & $(0.49,16,0.052)$ & $\ldots$ & $(0.51,13,0.075)$ \\
\hline$[\mathrm{Eu}$ II $/ \mathrm{Fe} \mathrm{I}]$ & $(0.49,16,0.052)$ & (2) & $(0.51,13,0.075)$ & $\ldots$ \\
\hline \multirow[t]{2}{*}{ [Ho II/Fe I] } & $(0.81,9,0.0080)$ & $(0.72,8,0.042)$ & $(0.36,6,0.48)$ & $(0.68,6,0.13)$ \\
\hline & [La II/Ti II $]$ & [La II/V II $]$ & [La II/Ti II] & [La II/V II $]$ \\
\hline [Eu II/Ti II] & $(0.69,16,0.0033)$ & & $(0.66,13,0.013)$ & \\
\hline [Eu II/V II] & $\ldots$ & $(0.73,16,0.0014)$ & $\ldots$ & $(0.80,13,0.0011)$ \\
\hline
\end{tabular}

Notes. Each set of data indicates $r, N$, and $P_{c}(r ; N)$. If two element ratios of a parent distribution are uncorrelated, the probability that a random sample of $N$ stars will yield a correlation coefficient $\geqslant|r|$ is given by $P_{c}(r ; N)$.

${ }^{\text {a }}$ Sneden et al. (2000).

of $[\mathrm{La} / \mathrm{Fe}]([\mathrm{Eu} / \mathrm{Fe}])$ observed in $\mathrm{M} 92$, would require $\Delta T_{\text {eff }} \approx$ $500 \mathrm{~K}(570 \mathrm{~K}),{ }^{7} \Delta \log g \approx 1.1(3.0), \Delta v_{t} \approx 4.5 \mathrm{~km} \mathrm{~s}^{-1}$-which is clearly non-physical, and $\Delta[\mathrm{M} / \mathrm{H}] \approx 1.2 \mathrm{dex}$. Uncertainties are not expected to be linear and are certainly correlated, but these estimates are illuminating. Furthermore, this scatter would be minimized when comparing ratios of La II or Eu II to Ti II or V II since these species all respond similarly to changes in the atmosphere; even so, the correlations between $[\mathrm{La} / \mathrm{Ti}, \mathrm{V}]$ and $[\mathrm{Eu} / \mathrm{Ti}, \mathrm{V}]$ are still highly significant (see Table 16). We conclude that it is extremely unlikely that random scatter in the photometry or relative model atmosphere parameters can account for the observed dispersion and correlation in $[\mathrm{La} / \mathrm{Fe}]$ and $[\mathrm{Eu} / \mathrm{Fe}]$.

In conclusion, several lines of evidence each point to intrinsic star-to-star dispersion in the heavy $n$-capture element abundances in the red giants we have studied in M92.

\footnotetext{
7 This corresponds to $\Delta(V-K) \approx 0.5 \mathrm{mag}$.
}

\subsection{Examining Correlations with Light-element Dispersion}

Does the $n$-capture element dispersion correlate with the lightelement dispersion? Figure 8 shows $[\mathrm{La} / \mathrm{Fe}]$ and $[\mathrm{Eu} / \mathrm{Fe}]$ as a function of $[\mathrm{C} / \mathrm{Fe}]$. Neither exhibits a significant correlation (see Table 16). Figure 8 also shows $[\mathrm{La} / \mathrm{Fe}]$ and $[\mathrm{Eu} / \mathrm{Fe}]$ as a function of $[\mathrm{N} / \mathrm{Fe}]$, and again there is no significant correlation. Sneden et al. (2000) derived $[\mathrm{Na} / \mathrm{Fe}]$ ratios for 13 and 12 stars whose $[\mathrm{La} / \mathrm{Fe}]$ and $[\mathrm{Eu} / \mathrm{Fe}]$ ratios we have derived. Figure 8 illustrates that no correlation exists between either $[\mathrm{La} / \mathrm{Fe}]$ or $[\mathrm{Eu} / \mathrm{Fe}]$ and $[\mathrm{Na} / \mathrm{Fe}]$.

Smith (2008) points out that in a limited number of GCs, including M92 and M15, the AGB stars with the highest $[\mathrm{Na} / \mathrm{Fe}]$ ratios are often those with the highest $[\mathrm{Ba} / \mathrm{Fe}]$ ratios. It is evident from Figures 5 and 6 that the four probable RHB/AGB stars we have analyzed in M92 often are among those with the highest $[\mathrm{Ba} / \mathrm{Fe}],[\mathrm{La} / \mathrm{Fe}],[\mathrm{Eu} / \mathrm{Fe}]$, and $[\mathrm{Ho} / \mathrm{Fe}]$ ratios. Several authors whose GC data were reexamined by Smith noted that 

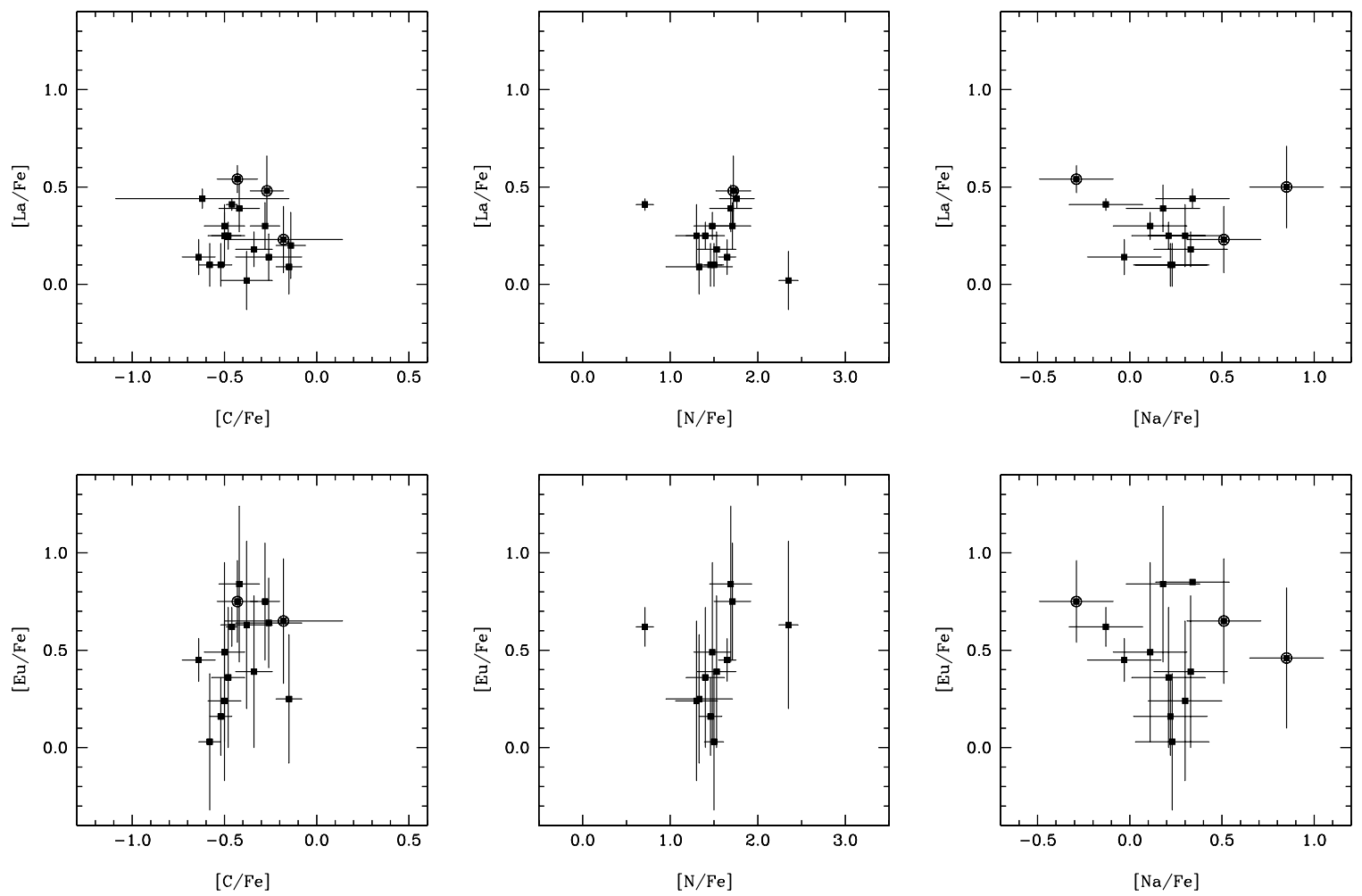

Figure 8. Comparison of the $[\mathrm{La} / \mathrm{Fe}]$ and $[\mathrm{Eu} / \mathrm{Fe}]$ ratios against $[\mathrm{C} / \mathrm{Fe}],[\mathrm{N} / \mathrm{Fe}]$, and $[\mathrm{Na} / \mathrm{Fe}]$. The $[\mathrm{Na} / \mathrm{Fe}]$ ratios are taken from Sneden et al. (2000). Open circles indicate probable AGB stars. None of these ratios exhibits a significant correlation. Only the internal (i.e., star-to-star) uncertainties are shown.

their $[\mathrm{Ba} / \mathrm{Fe}]$ ratios may be unreliable in the AGB stars. This could arise if the strong, saturated Ba II lines are formed (at least in part) in the chromospheric layers not accounted for in the models (Shetrone \& Keane 2000) or if the microturbulent velocity parameter derived from $\mathrm{Fe}_{\mathrm{I}}$ lines is not appropriate for the Ba II line-forming layers (Ivans et al. 2001). ${ }^{8}$ Both explanations are likely true to some extent and may apply to other analyses, and we regard the evidence for $s$-process selfenrichment in low-mass AGB stars as inconclusive.

Nevertheless, we conservatively reexamine the $n$-capture dispersion in M92 with the AGB stars excluded. Table 16 lists the correlation coefficients and probabilities for the remaining 15 stars. There is still no evidence that $[\mathrm{La} / \mathrm{Fe}]$ or $[\mathrm{Eu} / \mathrm{Fe}]$ correlate with $[\mathrm{C} / \mathrm{Fe}],[\mathrm{N} / \mathrm{Fe}]$, or $[\mathrm{Na} / \mathrm{Fe}]$. The correlations among the heavy $n$-capture elements are still significant, though generally less so because fewer stars are included. The [La II/Ti II] versus [Eu II/Ti II] and [La II/V II] versus [Eu II/V II] correlations are still highly significant.

In summary, these data indicate that the $n$-capture dispersion in M92 is robust and independent of the light-element dispersion.

\section{DISCUSSION}

We have demonstrated that the heavy $n$-capture abundances vary together relative to Fe in M92. What is the nucleosynthetic origin of the $n$-capture material, and how does this phenomenon relate to the $r$-process dispersion observed in M15? Other

8 Ivans et al. (2001) also note that their M5 [La/Eu] ratios are lower for the AGB stars than the RGB stars, $\langle[\mathrm{La} / \mathrm{Eu}]\rangle_{\mathrm{AGB}}=-0.46 \pm 0.05(\sigma=0.12)$ and $\langle[\mathrm{La} / \mathrm{Eu}]\rangle_{\mathrm{RGB}}=-0.37 \pm 0.05(\sigma=0.18)$. This is the opposite sense of what would be expected if the AGB stars contained a larger fraction of $s$-process material than the RGB stars. From this Ivans et al. conclude that the enhanced $[\mathrm{Ba} / \mathrm{Fe}]$ ratios in their AGB stars are not likely due to $s$-process enrichment. matters concerning the astrophysical mechanism(s) that lead to star-to-star dispersion are not so straightforward. In this section we discuss each of these matters.

\subsection{The r-process Abundance Pattern in M92}

Figure 9 shows the abundance distribution for the $Z \geqslant$ 39 elements in M92. ${ }^{9}$ In the top panel, we compare the M92 abundances to that of the S.S. $s$-process pattern (Sneden et al. 2008) and the $r$-process standard star CS 22892-052 (Sneden et al. 2003, 2009). The M92 abundances clearly resemble $r$-process nucleosynthesis more than $s$-process nucleosynthesis. In the bottom panel, the M92 abundances are compared with three metal-poor $r$-enriched field stars, CS 22892-052, HD 221170 (Ivans et al. 2006; Sneden et al. 2009), and HD 175305 (Roederer et al. 2010b). These three comparison stars have a range of heavy-element abundances that effectively bracket the mean $[\mathrm{Eu} / \mathrm{Fe}]$ ratio of M92, and Roederer et al. (2009, 2010a) demonstrated that the low $[\mathrm{Pb} / \mathrm{Eu}]$ ratios (or upper limits) in these three stars suggest that they contain no detectable trace of $s$-process material. Ba, Ce, and Nd in HD 221170 and HD 175305 are slightly higher than their abundances in CS 22892-052 when normalized to Eu, which Roederer et al. (2010a) argued to be a result of intrinsic variations in $r$-process nucleosynthesis, perhaps a result of different physical conditions at the nucleosynthesis site. M92 has an abundance pattern nearly identical to that of HD 175305, which has a similar $[\mathrm{Eu} / \mathrm{Fe}]$ ratio, $+0.35 \pm 0.15$, as what we

\footnotetext{
9 In an effort to detect weak lines of additional $n$-capture species, we have co-added the spectra of 21 individual stars on the RGB with $4730 \leqslant T_{\text {eff }} \leqslant$ $5080 \mathrm{~K}$ and $1.7 \leqslant \log g \leqslant 2.1$. This combined spectrum has $\mathrm{S} / \mathrm{N} \sim 270$ at 4000 A. Unfortunately, we are only able to detect one new line from this spectrum, Dy II 3944.68 ̊. From this spectrum we determine a mean $\log \epsilon(\mathrm{Dy} / \mathrm{La})=+0.25 \pm 0.3$. This value is shown in Figure 9 .
} 

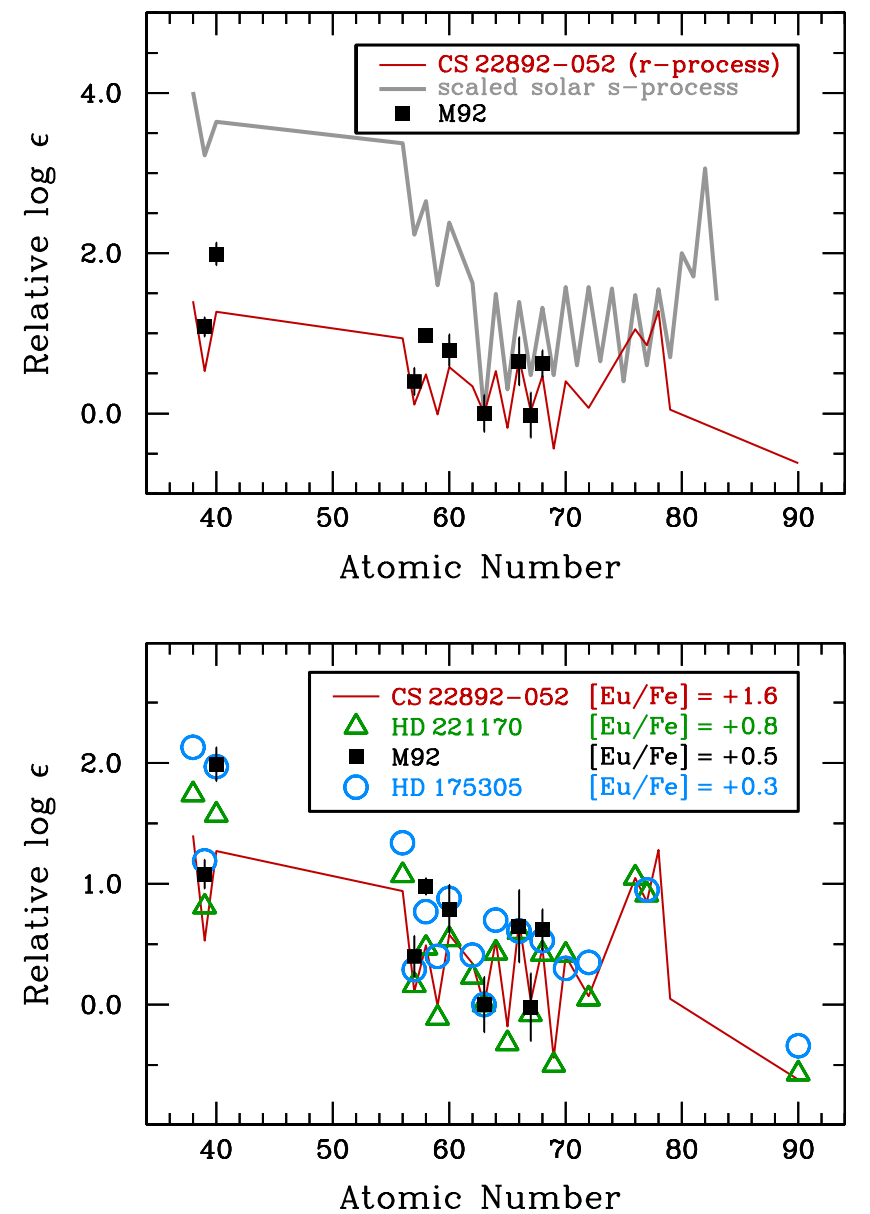

Figure 9. Top: comparison of the mean heavy-element abundances in M92 with the metal-poor $r$-process standard star CS 22892-052 and the scaled S.S. $s$-process pattern. The abundances are normalized to Eu. Bottom: comparison of the mean heavy-element abundances in M92 with three metal-poor field stars with differing levels of $r$-process enrichment. The abundances are normalized to Eu. The M92 abundance pattern is very similar to that in HD 175305 , which has a similar level of $[\mathrm{Eu} / \mathrm{Fe}]$ and similar ratios of $[\mathrm{Y} / \mathrm{Eu}]$ and $[\mathrm{Zr} / \mathrm{Eu}]$. Since all abundances are normalized to Eu, only the internal (i.e., star-to-star) uncertainties are shown.

(A color version of this figure is available in the online journal.)

have derived for M92. Other heavy-element abundances are similar between these two stars. Figure 9 implies that the heavy elements $(Z>56)$ in M92 originated in an $r$-process.

At low metallicity, the $s$-process produces large $[\mathrm{Pb} / \mathrm{Eu}]$ ratios due to the high ratio of neutrons to $\mathrm{Fe}$-group seed nuclei (e.g., Clayton 1988; Gallino et al. 1998), so [Pb/Eu] is a good diagnostic of $s$-process material in metal-poor stars. Our spectra just miss the Pb I line at $4057 \AA$. Shetrone et al. (2001) obtained high resolution blue spectra with Keck HIRES of two stars in M92, III-13 and III-65. From these spectra (M. Shetrone 2011, private communication) we use the Eu II $4129 \AA$ line (detected) and the $\mathrm{Pb}$ I $4057 \AA$ line (not detected) to derive an approximate upper limit on $\mathrm{Pb},[\mathrm{Pb} / \mathrm{Eu}] \lesssim+0.3$. This is low enough (Roederer et al. 2010a) to rule out contributions from low-metallicity intermediate-mass AGB stars to the gas from which the M92 stars formed, reinforcing our assertion that the $Z>56$ material in M92 originated only in an $r$-process.

Nucleosynthesis of the Sr-Y-Zr group of elements is more complex. While only true $n$-capture processes can produce elements heavier than the $A \simeq 130$ peak in significant quantities, several other charged-particle reaction mechanisms like the $v p$ process or $\alpha$-rich freezeout may also contribute to-if

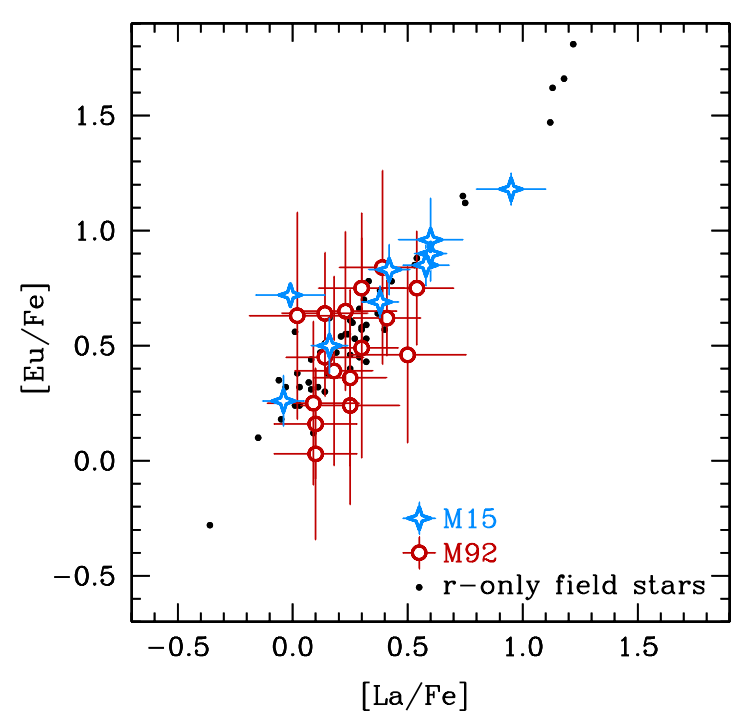

Figure 10. Comparison of the $[\mathrm{Eu} / \mathrm{Fe}]$ and $[\mathrm{La} / \mathrm{Fe}]$ ratios (and total uncertainties) in M92 with those in M15 (Sobeck et al. 2011) and a sample of field stars whose only enrichment in $\mathrm{La}$ and $\mathrm{Eu}$ has come from $r$-process nucleosynthesis (Roederer et al. 2010a).

(A color version of this figure is available in the online journal.)

not dominate production of-the lighter Sr-Y-Zr group (e.g., Woosley et al. 1994, Freiburghaus et al. 1999, Fröhlich et al. 2006, Arcones \& Montes 2011, Farouqi et al. 2010). In M92, these elements display a dispersion similar to that of the Fe-group elements. The predictable nature of the $[\mathrm{Y} / \mathrm{Eu}]$ or $[\mathrm{Zr} / \mathrm{Eu}]$ ratios based on the $[\mathrm{Eu} / \mathrm{Fe}]$ ratio (inferred from Figure 9) is therefore a consequence of rather similar $[\mathrm{Y} / \mathrm{Fe}]$ and $[\mathrm{Zr} / \mathrm{Fe}]$ ratios. The fact that the heavy (La-Er) elements in M92 have a larger dispersion than $\mathrm{Y}$ or $\mathrm{Zr}$ implies that these groups are produced mainly by different nucleosynthetic mechanisms.

\subsection{Comparison with Globular Cluster M15}

M15 is more massive than M92 $\left(7.6 \times 10^{5} M_{\odot}\right.$ and $3.1 \times 10^{5} M_{\odot}$, respectively, assuming $\left.M / L_{V}=2 M_{\odot} / L_{\odot}\right)$, but both have nearly identical metallicities and ages. Sneden et al. (1997) identified a significant dispersion in [Ba/Fe] in M15. Sneden et al. (2000), Otsuki et al. (2006), and Sobeck et al. (2011) confirmed this result and extended it to all $Z \geqslant$ 56 elements that have been studied in M15. These studies have shown that the heavy elements in M15 are produced by $r$-process nucleosynthesis, and there are no hints of $s$-process contamination. M15 is the only other GC where dispersion of $r$-process material has been reported.

Figure 10 compares $[\mathrm{La} / \mathrm{Fe}]$ and $[\mathrm{Eu} / \mathrm{Fe}]$ in $\mathrm{M} 15$ and M92. Both ratios vary over a wide range in each GC $(-0.1<$ $[\mathrm{La} / \mathrm{Fe}]<+0.9$ and $+0.2<[\mathrm{Eu} / \mathrm{Fe}]<+1.2$ in M15, $0.0<[\mathrm{La} / \mathrm{Fe}]<+0.6$ and $0.0<[\mathrm{Eu} / \mathrm{Fe}]<+0.9$ in M92, but note that the ranges of M15 ratios include a systematic offset of 0.35 dex between the RGB and RHB stars examined by Sobeck et al. 2011). [ $\mathrm{La} / \mathrm{Fe}]$ and $[\mathrm{Eu} / \mathrm{Fe}]$ correlate with each other in both M15 and M92, similar to the correlation found in metalpoor halo field stars enriched by $r$-process nucleosynthesis. Armosky et al. (1994) and Sneden et al. (2000) showed that the mean $[\mathrm{Ba} / \mathrm{Fe}]$ ratios are higher in M15 than in M92, but the $[\mathrm{Ba} / \mathrm{Eu}]$ ratios in both GCs reflect only $r$-process nucleosynthesis. Based on comparisons between our results and previous studies (Section 3.5), we confirm that the mean $[\mathrm{Eu} / \mathrm{Fe}]$ ratio in M92 is lower than that in M15. These data do not suggest why 
M15 contains a larger mean $r$-process overabundance relative to M92. Nevertheless, it is clear that the stars in both M15 and M92 exhibit a range of $r$-process abundances.

Sneden et al. (1997) looked for and found no correlation between the light (among C, N, O, Na, Mg, and Al) and heavyelement dispersion (among Ba and Eu) in M15. We confirm that M92 behaves similarly. The data imply that the $r$-process dispersion in M15 and M92 was imprinted in the gas from which all present-day GC stars, including those of the second generation, were formed.

\subsection{Explanations for the Origin of the r-process Dispersion}

Some mechanism(s) must account for the ability of M15 and M92 to arrive at a homogeneous set of $\mathrm{Ca}$ - and Fe-group abundances and an inhomogeneous set of heavy $n$-capture abundances before the source of the light-element dispersion becomes an active participant in the chemical evolution of these GCs. Since the $r$-process dispersion is also present in later generations of stars, some mechanism(s) must also preserve this inhomogeneity over long ( $\gtrsim 10 \mathrm{Myr}$ ) timescales (see also D'Orazi et al. 2010).

Despite the time that has passed since Sneden et al. (1997) first reported an $r$-process dispersion in M15, we are aware of no published attempts to explain this phenomenon. Variations in the $[\mathrm{La} / \mathrm{Eu}]$ ratios-observed in $r$-only field stars and M92-suggest that dilution of the yields from rare but identical $r$-process events cannot alone account for the inhomogeneous distribution of $r$-process material. Both M15 and M92 are on moderately eccentric (but unrelated) Galactic orbits, and each is currently located near its apogalactic radius (approximately 10 kpc; Dinescu et al. 1999). Other massive GCs (e.g., $\omega$ Cen) exhibit a complex variety of abundance patterns; these GCs likely formed in much larger parent systems, since disrupted by the Milky Way, that were capable of driving chemical evolution within themselves. Neither M15 nor M92 has been associated with tidal debris from a dwarf galaxy or stellar streams in the Galactic halo (e.g., Smith et al. 2009), and neither CMD exhibits multiple main sequences or subgiant branches. We conclude that there is no convincing explanation at present for the observed $r$-process dispersion in M15 and M92.

\section{CONCLUSIONS}

We have obtained new high $\mathrm{S} / \mathrm{N}$ spectra covering 3850-4050 A for 19 stars in the metal-poor GC M92 using the Hydra spectrograph on the WIYN Telescope. We perform a detailed differential abundance analysis and quantify the chemical homogeneity in M92 for 21 species of 19 elements from carbon to erbium. Our main results are summarized as follows.

1. These stars are chemically homogeneous at the level of $0.07-0.16$ dex for $\mathrm{Sc}, \mathrm{Ti}, \mathrm{V}, \mathrm{Cr}, \mathrm{Fe}, \mathrm{Co}, \mathrm{Ni}, \mathrm{Y}$, and $\mathrm{Zr}$. The absolute metallicity and $[\mathrm{X} / \mathrm{H}]$ ratios should be treated with caution, but the ratios among metals are quite robust.

2. The heavy $n$-capture elements $\mathrm{La}, \mathrm{Eu}$, and $\mathrm{Ho}$ are not chemically homogeneous throughout these 19 stars in M92. The $[\mathrm{La} / \mathrm{Fe}],[\mathrm{Eu} / \mathrm{Fe}]$, and $[\mathrm{Ho} / \mathrm{Fe}]$ ratios have dispersions of $0.17-0.28$ dex and span ranges of $0.5-0.8$ dex (a factor of 3-6). This dispersion is not due to observational uncertainty since these ratios correlate with each other and with the $[\mathrm{Ba} / \mathrm{Fe}]$ ratios derived by Sneden et al. (2000).

3. The elements $\mathrm{Y}$ and $\mathrm{Zr}$ show dispersion similar to that of the Fe-group and less than that of $\mathrm{Ba}, \mathrm{La}, \mathrm{Eu}$, and Ho. This suggests that that the $\mathrm{Y}$ and $\mathrm{Zr}$ were not formed primarily by $r$-process nucleosynthesis and were more uniformly mixed at the time of star formation.

4. The heaviest elements originate in $r$-process nucleosynthesis without contributions from the $s$-process. The $r$-process dispersion does not correlate with the light-element dispersion $(\mathrm{C}, \mathrm{N}$, and $\mathrm{Na})$, indicating that the $r$-process dispersion was present in the gas throughout star formation.

5. The $r$-process dispersion in M92 is similar-but not identical to-that observed previously in the massive, metalpoor GC M15 (e.g., Sneden et al. 1997). Both GCs show unmistakable star-to-star dispersion of $r$-process material relative to $\mathrm{Fe}$. The dispersion in M15 is larger and the mean $r$-process level is higher in M15 than in M92. Sneden et al. demonstrated that the $r$-process dispersion in M15, like M92, also does not correlate with the light-element dispersion.

There are at least two (perhaps several; Roederer 2011) massive, metal-poor Milky Way GCs that formed from material with inhomogeneous distributions of $r$-process material. At present there exists no explanation for the astrophysical mechanism(s) responsible for this phenomenon. Attempts to understand and incorporate this into the rapidly evolving theory of GC formation and evolution will surely prove rewarding.

We thank C. I. Johnson and C. Pilachowski for their helpful advice and generous assistance in preparing for our observing run, M. Shetrone for sharing his M92 spectra, J. A. Johnson for useful discussions, A. Marino and J. Sobeck for conveying abundance results in advance of publication, and the referee for a careful review of our work. We are extremely grateful to M. Spite and the "First Stars" team, J. A. Johnson, as well as G. Preston, S. Shectman, and I. Thompson for permitting us to compare portions of their spectra with our own data. This research has made use of the NASA Astrophysics Data System (ADS), the NIST Atomic Spectra Database, and the Two Micron All Sky Survey, which is a joint project of the University of Massachusetts and the Infrared Processing and Analysis Center/ California Institute of Technology, funded by the National Aeronautics and Space Administration and the National Science Foundation. I.U.R. is supported by the Carnegie Institution of Washington through the Carnegie Observatories Fellowship. C.S. is supported by the U.S. National Science Foundation (grant AST 09-08978).

Facilities: WIYN (Hydra)

\section{APPENDIX}

\section{THE METALLICITY OF M92}

Our absolute metallicity for M92 is anchored to the Fe I abundance in XII-8, the reference star used in our analysis. The metallicities of all other M92 stars in our analysis have been computed differentially with respect to XII-8. The mean metallicity derived from 19 RGB stars, $[\mathrm{Fe} \mathrm{I} / \mathrm{H}]=-2.70 \pm$ 0.03 , is lower by more than a factor of two than that derived from 33 RGB stars by Sneden et al. (2000), [Fe I/H] $=-2.34 \pm$ 0.01 . Based on equivalent width (EW) measurements of Fe I or Fe II lines from high resolution spectra, numerous studies over the last 20 years have derived metallicities ranging from $-2.4<$ $[\mathrm{Fe} / \mathrm{H}]<-2.1$ for M92, though Peterson et al. (1990) and King et al. (1998) have presented evidence for $[\mathrm{Fe} / \mathrm{H}] \lesssim-2.5$ in M92.

There are three significant differences between our study and Sneden et al. (2000) that in principle may account for portions 


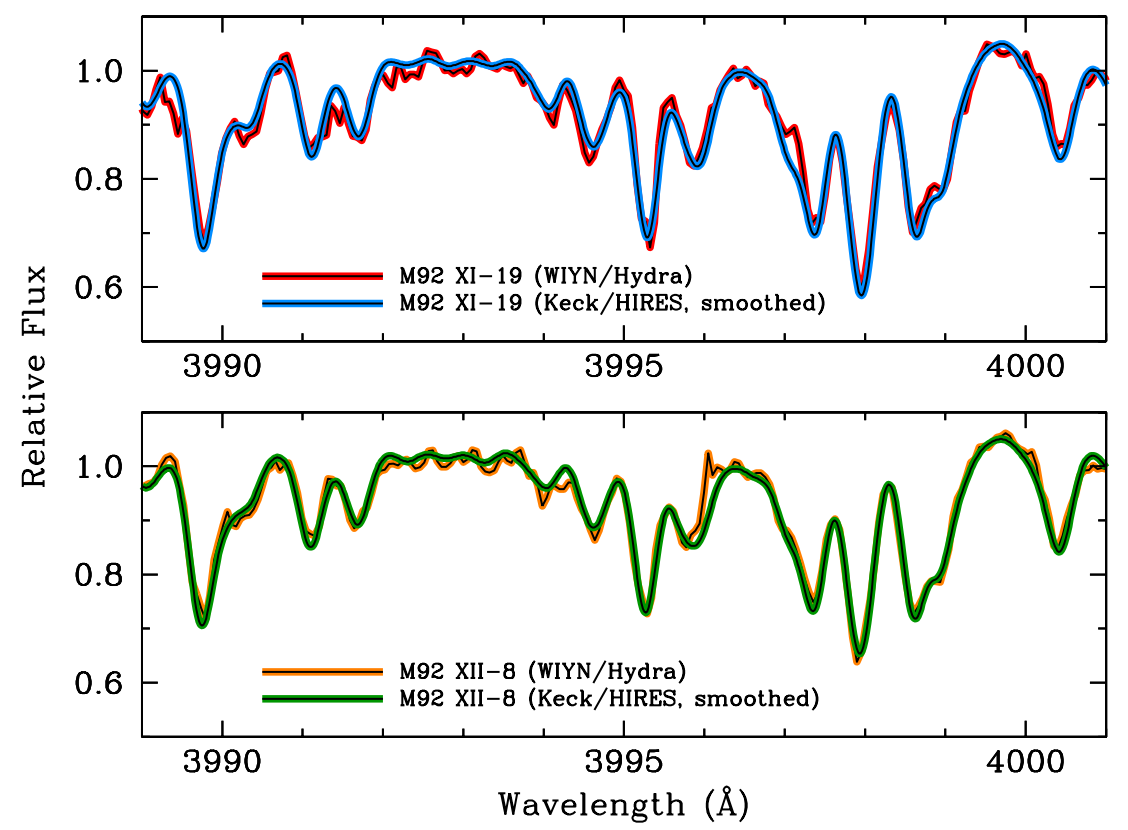

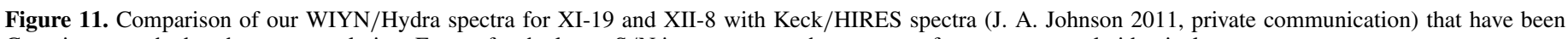
Gaussian smoothed to the same resolution. Except for the lower S/N in our spectra, the two sets of spectra are nearly identical.

(A color version of this figure is available in the online journal.)

of this offset, including (1) different laboratory sources for the $\mathrm{Fe}_{\mathrm{I}} \log (g f)$ values and different sets of $\mathrm{Fe}$ I lines available for analysis; (2) different grids of model atmospheres (we use the most recent set of MARCS $\alpha$-enhanced models, Gustafsson et al. 2008, while Sneden et al. used the Gustafsson et al. 1975 set available at the time); and (3) different versions of the MOOG code, with the most notable difference being the explicit calculation of the Rayleigh scattering contribution to the blue continuous opacity as described in Sobeck et al. (2011). We check each of these effects below.

O'Brian et al. (1991), our preferred source for Fe I $\log (g f)$ values, did not report $\log (g f)$ values for five of the seven lines covered by Sneden et al.; for the remaining two lines, the O'Brian et al. values are higher by 0.12 and 0.19 dex. Naively extrapolating these offsets suggests that the Sneden et al. Fe I abundance could be lower by $0.1-0.2$ dex on the O'Brian et al. $\log (g f)$ scale. Rederiving the $\mathrm{Fe}_{\mathrm{I}}$ abundance of XII-8 using the Sneden et al. EWs and the MARCS model used in the present study (which accounts for differences in model parameters and grids) decreases the abundance by 0.12 dex. Rederiving the XII- 8 abundance from the two versions of MOOG decreases the Sneden et al. abundance by 0.01 dex. Together, these effects can produce a decrease of $\sim 0.2-0.3$ dex in the abundance derived by Sneden et al. We have derived $[\mathrm{Fe} / \mathrm{H}]$ lower by $0.55 \mathrm{dex}$ for XII-8, so these effects can account for about half of the discrepancy. The standard deviation of the nine Fe I lines we have examined in XII-8 is 0.18 dex and Sneden et al. produced a standard deviation of 0.25 dex from four Fe I lines. This could, in principle, account for another significant portion of the discrepancy.

In Section 3.4 we found [Ti II/Fe I] to be higher than [Ti I/Fe I] by $\sim 0.5 \mathrm{dex}$, and in Section 3.5 we found that our [X II/Fe I] ratios were higher by $0.2-0.6 \mathrm{dex}$ than had been found in previous studies of VII-18. We have not forced Fe (or Ti) ionization equilibrium when deriving our atmospheric parameters. The singly ionized species are the dominant ones for Fe-group elements in these stellar atmospheres, and neglecting to account for departures from LTE in our analysis would tend to underestimate the abundance of the neutral species. By this reasoning it is plausible that our $[\mathrm{Fe} \mathrm{I} / \mathrm{H}]$ abundances have been underestimated by several tenths of a dex.

Adopting a different photometric temperature scale would not have altered our results significantly. For these M92 stars, the Alonso et al. (1999) $V-K$ scale predicts no significant difference for stars with $T_{\text {eff }}>4650 \mathrm{~K}$, but for the cooler giants it predicts temperatures systematically lower by $60-130 \mathrm{~K}$. For XII-8, Sneden et al. (who used $B-V$ versus $T_{\text {eff }}$ relations derived by Carbon et al. 1982) derived $T_{\text {eff }}=4490 \mathrm{~K}$. Using the Alonso et al. scale we would derive $4380 \mathrm{~K}$, and using the Ramírez \& Meléndez (2005b) scale we have derived $4450 \mathrm{~K}$. For XII-8, our tests indicate that $\Delta T_{\text {eff }}= \pm 100 \mathrm{~K}$ translates to $\Delta[\mathrm{Fe} \mathrm{I} / \mathrm{H}]= \pm 0.10$ dex. This corresponds to $[\mathrm{Fe} / \mathrm{H}]$ differences of +0.04 dex and -0.08 dex with respect to Sneden et al. and the Alonso et al. scale, respectively. Thus, adopting any of these three temperature scales would have produced similar metallicity results.

We have compared our WIYN/Hydra spectra for two stars, XI-19 and XII-8 (our reference star), with spectra of these two stars obtained by J. A. Johnson (2011, private communication) using Keck/HIRES. Figure 11 illustrates this comparison for a representative wavelength range. We have smoothed the HIRES spectra down to our Hydra resolution. Our spectra have lower $\mathrm{S} / \mathrm{N}$, but otherwise the spectra for these two stars are essentially identical. This gives us confidence that we have not made serious errors during the extraction procedure (e.g., poor subtraction of sky or scattered light from the image frames).

Recently, others have found a similarly low metallicity for stars in M15: $[\mathrm{Fe} \mathrm{I} / \mathrm{H}]=-2.66$ and $[\mathrm{Fe} \mathrm{II} / \mathrm{H}]=-2.60$ from six RHB stars (Preston et al. 2006), [Fe I/H] $=-2.69$ and $[\mathrm{Fe} \mathrm{II} / \mathrm{H}]$ $=-2.64$ from the same six RHB stars or $[\mathrm{Fe} \mathrm{I} / \mathrm{H}]=-2.56$ and $[\mathrm{Fe} \mathrm{II} / \mathrm{H}]=-2.53$ from three RGB stars (Sobeck et al. 2011). Tests conducted by Preston et al. and Sobeck et al. indicate that the persistent metallicity offset between the RGB and RHB stars and the metallicity offset between the RGB stars studied by them 


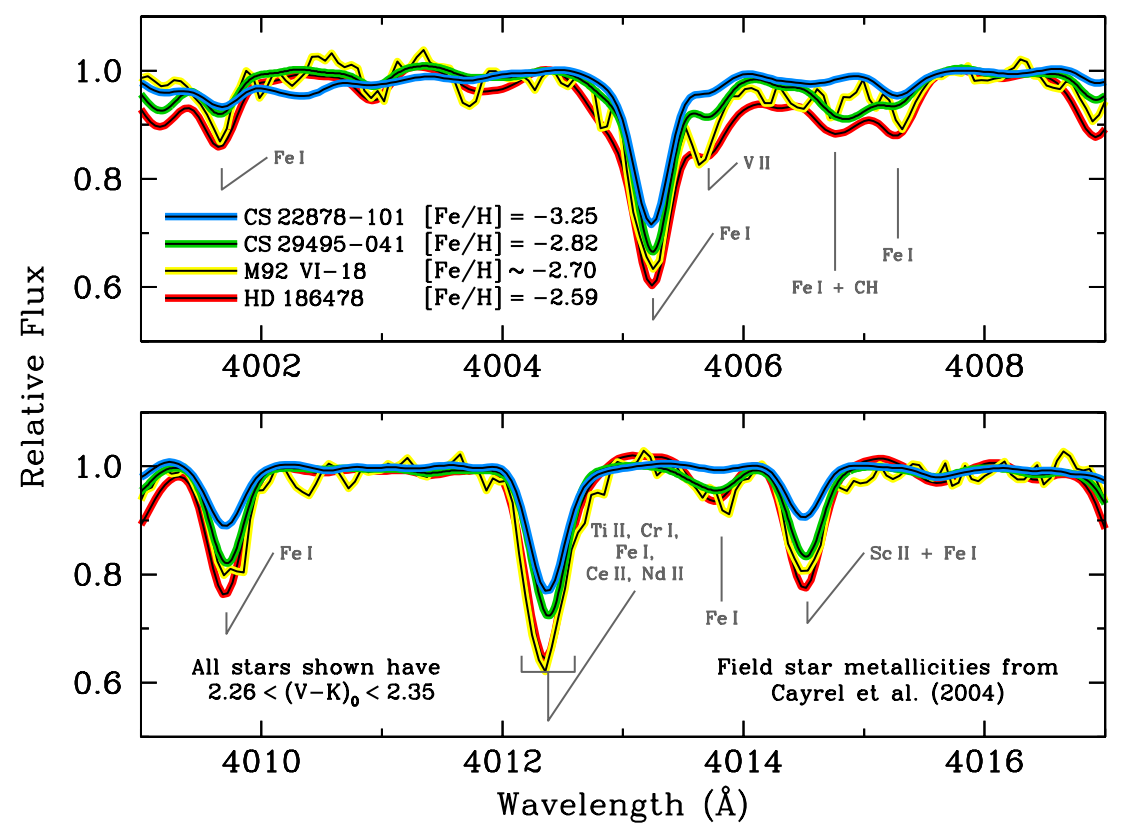

Figure 12. Comparison of spectra for four stars with very similar colors but differing metallicities. The spectrum of VI-18 was obtained in our study, the spectrum of CS 29495-041 was obtained as part of the "First Stars" project using UVES on the VLT (M. Spite 2010, private communication), and the spectra of CS 22878-101 and HD 186478 were obtained in follow-up to the "HK Survey" using MIKE on the Magellan-Clay Telescope. All spectra have been Gaussian smoothed to the resolution of VI-18. Note that most absorption features in this wavelength range are due to Fe-group elements.

(A color version of this figure is available in the online journal.)

and Sneden et al. $(1997,2000)$ are not a result of the choice of atmospheric parameters, line lists, model atmosphere grids, or recent upgrades to MOOG. The offset between the cool RGB stars and the warm RGB/RHB stars in our M92 study runs in the opposite direction. We note that five of the six RHB stars in M15 studied by Sobeck et al. are significantly warmer than our warmest star in M92, so considering the $T_{\text {eff }}-[\mathrm{Fe} / \mathrm{H}]$ slopes in this simple manner may not afford a fair comparison

Finally, in Figure 12 we present an empirical verification that our metallicities are consistent with those derived for other metal-poor field RGB stars. All four stars shown in Figure 12 have very similar colors, and in all four cases the reddening is small, $E(B-V)<0.1$. Most of the absorption lines in the wavelength region shown are due to $\mathrm{Fe}$-group species. The metallicities listed for CS 22878-101, CS 29495-041, and HD 186478 are reported directly from Cayrel et al. (2004), whose abundances are frequently used as abundance standards for low-metallicity field RGB stars in the solar neighborhood. (The metallicities derived by McWilliam et al. 1995 for these stars are very similar.) The metallicity of VI- 18 inferred from the spectra of these other three stars, $[\mathrm{Fe} / \mathrm{H}] \approx-2.7$, agrees well with the metallicity we have derived in our own analysis, $[\mathrm{Fe} / \mathrm{H}]=-2.78 \pm 0.06(\sigma=0.18)$. (VI-18 is chosen for comparison because it has a color very similar to several stars in the Cayrel et al. sample with higher and lower metallicities.) Performing the same tests on these stars as described above (comparing $\log (g f)$ values, rederiving $[\mathrm{Fe} \mathrm{I} / \mathrm{H}]$ using the Cayrel et al. EWs and atmospheric parameters but our analysis tools) only leads to a lowering of their $[\mathrm{Fe} / \mathrm{H}]$ by $0.07 \mathrm{dex}$. Our derived metallicities thus appear reasonable when compared with metalpoor field RGB stars.

\section{REFERENCES}

Alonso, A., Arribas, S., \& Martínez-Roger, C. 1999, A\&AS, 139, 335 Arcones, A., \& Montes, F. 2011, ApJ, 731, 5
Armosky, B. J., Sneden, C., Langer, G. E., \& Kraft, R. P. 1994, AJ, 108, 1364 Asplund, M., Grevesse, N., Sauval, A. J., \& Scott, P. 2009, ARA\&A, 47, 481 Barden, S. C., \& Armandroff, T. 1995, Proc. SPIE, 2476, 56

Bergemann, M. 2011, MNRAS, 413, 2184

Bershady, M., et al. 2008, Proc. SPIE, 7014, 70140H

Bevington, P. R., \& Robinson, D. K. 2003, Data Reduction and Error Analysis for the Physical Sciences (3rd ed., Boston, MA: McGraw-Hill), 197

Biémont, E., Grevesse, N., Faires, L. M., Marsden, G., \& Lawler, J. E. 1989, A\&A, 209, 391

Blackwell, D. E., Petford, A. D., Shallis, M. J., \& Leggett, S. 1982, MNRAS, 199, 21

Blackwell-Whitehead, R., \& Bergemann, M. 2007, A\&A, 472, L43

Bonifacio, P., et al. 2009, A\&A, 501, 519

Booth, A. J., Blackwell, D. E., Petford, A. D., \& Shallis, M. J. 1984, MNRAS, 208, 147

Buonanno, R., Buscema, G., Corsi, C. E., Iannicola, G., Smriglio, F., \& Pecci, F. F. 1983 , A\&AS, 53,1

Carbon, D. F., Romanishin, W., Langer, G. E., Butler, D., Kemper, E., Trefzger, C. F., Kraft, R. P., \& Suntzeff, N. B. 1982, ApJS, 49, 207

Carretta, E., et al. 2010a, A\&A, 520, A95

Carretta, E., et al. 2010b, ApJ, 722, L1

Cayrel, R., et al. 2004, A\&A, 416, 1117

Clayton, D. D. 1988, MNRAS, 234, 1

Cohen, J. G. 1979, ApJ, 231, 751

Cohen, J. G., Kirby, E. N., Simon, J. D., \& Geha, M. 2010, ApJ, 725, 288

Cohen, J. G., \& McCarthy, J. K. 1997, AJ, 113, 1353

Cohen, J. G., et al. 2004, ApJ, 612, 1107

Cudworth, K. M. 1976, AJ, 81, 975

Den Hartog, E. A., Lawler, J. E., Sneden, C., \& Cowan, J. J. 2003, ApJS, 148, 543

Dinescu, D. I., Girard, T. M., \& van Altena, W. F. 1999, AJ, 117, 1792

D’Orazi, V., Gratton, R., Lucatello, S., Carretta, E., Bragaglia, A., \& Marino, A. F. 2010, ApJ, 719, L213

Drukier, G. A., Cohn, H. N., Lugger, P. M., Slavin, S. D., Berrington, R. C., \& Murphy, B. W. 2007, AJ, 133, 1041

Farouqi, K., Kratz, K.-L., Pfeiffer, B., Rauscher, T., Thielemann, F.-K., \& Truran, J. W. 2010, ApJ, 712, 1359

Freiburghaus, C., Rembges, J.-F., Rauscher, T., Kolbe, E., Thielemann, F.-K., Kratz, K.-L., Pfeiffer, B., \& Cowan, J. J. 1999, ApJ, 516, 381

Fröhlich, C., Martínez-Pinedo, G., Liebendörfer, M., Thielemann, F.-K., Bravo, E., Hix, W. R., Langanke, K., \& Zinner, N. T. 2006, Phys. Rev. Lett., 96, 142502 
Gallino, R., Arlandini, C., Busso, M., Lugaro, M., Travaglio, C., Straniero, O., Chieffi, A., \& Limongi, M. 1998, ApJ, 497, 388

Gratton, R. G., Carretta, E., \& Castelli, F. 1996, A\&A, 314, 191

Grevesse, N., Blackwell, D. E., \& Petford, A. D. 1989, A\&A, 208, 157

Gustafsson, B., Bell, R. A., Eriksson, K., \& Nordlund, A. 1975, A\&A, 42, 407

Gustafsson, B., Edvardsson, B., Eriksson, K., Jørgensen, U. G., Nordlund, Å., \& Plez, B. 2008, A\&A, 486, 951

Hannaford, P., Lowe, R. M., Grevesse, N., Biémont, E., \& Whaling, W. 1982, ApJ, 261, 736

Harris, W. E. 1996, AJ, 112, 1487

Ivans, I. I., Kraft, R. P., Sneden, C., Smith, G. H., Rich, R. M., \& Shetrone, M. 2001, AJ, 122, 1438

Ivans, I. I., Simmerer, J., Sneden, C., Lawler, J. E., Cowan, J. J., Gallino, R., \& Bisterzo, S. 2006, ApJ, 645, 613

Ivans, I. I., Sneden, C., Kraft, R. P., Suntzeff, N. B., Smith, V. V., Langer, G. E., \& Fulbright, J. P. 1999, AJ, 118, 1273

Johnson, C. I., \& Pilachowski, C. A. 2010, ApJ, 722, 1373

Johnson, J. A. 2002, ApJS, 139, 219

King, J. R., Stephens, A., Boesgaard, A. M., \& Deliyannis, C. 1998, AJ, 115 , 666

Kurucz, R. L., \& Bell, B. 1995, Kurucz CD-ROM (Cambridge, MA: Smithsonian Astrophysical Observatory)

Lai, D. K., Bolte, M., Johnson, J. A., Lucatello, S., Heger, A., \& Woosley, S. E. 2008, ApJ, 681, 1524

Langer, G. E., Fischer, D., Sneden, C., \& Bolte, M. 1998, AJ, 115, 685

Lawler, J. E., Bonvallet, G., \& Sneden, C. 2001a, ApJ, 556, 452

Lawler, J. E., \& Dakin, J. T. 1989, J. Opt. Soc. Am., 6, 1457

Lawler, J. E., Sneden, C., \& Cowan, J. J. 2004, ApJ, 604, 850

Lawler, J. E., Sneden, C., Cowan, J. J., Ivans, I. I., \& Den Hartog, E. A. 2009, ApJS, 182, 51

Lawler, J. E., Sneden, C., Cowan, J. J., Wyart, J.-F., Ivans, I. I., Sobeck, J. S., Stockett, M. H., \& Den Hartog, E. A. 2008, ApJS, 178, 71

Lawler, J. E., Wickliffe, M. E., den Hartog, E. A., \& Sneden, C. 2001b, ApJ, 563,1075

Lupton, R., Gunn, J. E., \& Griffin, R. F. 1985, in IAU Symp. 113, Dynamics of Star Clusters, ed. J. Goodman \& P. Hut (Cambridge: Cambridge Univ. Press), 19

Malcheva, G., Blagoev, K., Mayo, R., Ortiz, M., Xu, H. L., Svanberg, S., Quinet, P., \& Biémont, E. 2006, MNRAS, 367, 754

Marino, A., et al. 2011, A\&A, in press (arXiv:1105.1523)

Marino, A. F., Milone, A. P., Piotto, G., Villanova, S., Bedin, L. R., Bellini, A., \& Renzini, A. 2009, A\&A, 505, 1099

McCall, M. L. 2004, AJ, 128, 2144

McWilliam, A., Preston, G. W., Sneden, C., \& Searle, L. 1995, AJ, 109, 2757

Mészáros, S., Dupree, A. K., \& Szalai, T. 2009, AJ, 137, 4282

Nitz, D. E., Kunau, A. E., Wilson, K. L., \& Lentz, L. R. 1999, ApJS, 122, 557

Norris, J., \& Zinn, R. 1977, ApJ, 215, 74

O’Brian, T. R., \& Lawler, J. E. 1991, Phys. Rev. A, 44, 7134

O’Brian, T. R., Wickliffe, M. E., Lawler, J. E., Whaling, W., \& Brault, J. W. 1991, J. Opt. Soc. Am., 8, 1185

Otsuki, K., Honda, S., Aoki, W., Kajino, T., \& Mathews, G. J. 2006, ApJ, 641, L117

Peterson, C. J., \& Reed, B. C. 1987, PASP, 99, 20

Peterson, R. C., Kurucz, R. L., \& Carney, B. W. 1990, ApJ, 350, 173

Pickering, J. C., Thorne, A. P., \& Perez, R. 2002, ApJS, 138, 247

Pilachowski, C. A., Sneden, C., Kraft, R. P., Harmer, D., \& Willmarth, D. 2000, AJ, 119, 2895

Pont, F., Mayor, M., Turon, C., \& Vandenberg, D. A. 1998, A\&A, 329, 87
Preston, G. W., Sneden, C., Thompson, I. B., Shectman, S. A., \& Burley, G. S. 2006, AJ, 132, 85

Pryor, C., \& Meylan, G. 1993, in ASP Conf. Ser. 50, Structure and Dynamics of Globular Clusters, ed. S. G. Djorgovski \& G. Meylan (San Francisco, CA: ASP), 357

Ramírez, I., \& Meléndez, J. 2005a, ApJ, 626, 446

Ramírez, I., \& Meléndez, J. 2005b, ApJ, 626, 465

Reed, B. C., Hesser, J. E., \& Shawl, S. J. 1988, PASP, 100, 545

Rees, R. F., Jr. 1992, AJ, 103, 1573

Roederer, I. U. 2011, ApJ, 732, L17

Roederer, I. U., Cowan, J. J., Karakas, A. I., Kratz, K.-L., Lugaro, M., Simmerer, J., Farouqi, K., \& Sneden, C. 2010a, ApJ, 724, 975

Roederer, I. U., Kratz, K.-L., Frebel, A., Christlieb, N., Pfeiffer, B., Cowan, J. J., \& Sneden, C. 2009, ApJ, 698, 1963

Roederer, I. U., Sneden, C., Thompson, I. B., Preston, G. W., \& Shectman, S. A. 2010b, ApJ, 711, 573

Sadakane, K., Arimoto, N., Ikuta, C., Aoki, W., Jablonka, P., \& Tajitsu, A. 2004, PASJ, 56, 1041

Sandage, A. 1969, ApJ, 157, 515

Sandage, A., \& Walker, M. F. 1966, ApJ, 143, 313

Schlegel, D. J., Finkbeiner, D. P., \& Davis, M. 1998, ApJ, 500, 525

Shetrone, M. D. 1996, AJ, 112, 1517

Shetrone, M. D., Bolte, M., \& Stetson, P. B. 1998, AJ, 115, 1888

Shetrone, M. D., Côté, P., \& Sargent, W. L. W. 2001, ApJ, 548, 592

Shetrone, M. D., \& Keane, M. J. 2000, AJ, 119, 840

Skrutskie, M. F., et al. 2006, AJ, 131, 1163

Smith, G. H. 2008, PASP, 120, 952

Smith, M. C., et al. 2009, MNRAS, 399, 1223

Smith, V. V., Suntzeff, N. B., Cunha, K., Gallino, R., Busso, M., Lambert, D. L., \& Straniero, O. 2000, AJ, 119, 1239

Sneden, C., Cowan, J. J., \& Gallino, R. 2008, ARA\&A, 46, 241

Sneden, C., Kraft, R. P., Prosser, C. F., \& Langer, G. E. 1991, AJ, 102, 2001

Sneden, C., Kraft, R. P., Shetrone, M. D., Smith, G. H., Langer, G. E., \& Prosser, C. F. 1997, AJ, 114, 1964

Sneden, C., Lawler, J. E., Cowan, J. J., Ivans, I. I., \& Den Hartog, E. A. 2009, ApJS, 182, 80

Sneden, C., Pilachowski, C. A., \& Kraft, R. P. 2000, AJ, 120, 1351

Sneden, C., et al. 2003, ApJ, 591, 936

Sneden, C. A. 1973, PhD thesis, Univ. Texas at Austin

Sobeck, J. S., Lawler, J. E., \& Sneden, C. 2007, ApJ, 667, 1267

Sobeck, J. S., et al. 2011, AJ, 141, 175

Soderberg, A. M., Pilachowski, C. A., Barden, S. C., Willmarth, D., \& Sneden, C. 1999, PASP, 111, 1233

Strom, S. E., \& Strom, K. M. 1971, A\&A, 14, 111

Tucholke, H.-J., Scholz, R.-D., \& Brosche, P. 1996, A\&A, 312, 74

van den Bergh, S., Morbey, C., \& Pazder, J. 1991, ApJ, 375, 594

Villanova, S., Geisler, D., \& Piotto, G. 2010, ApJ, 722, L18

Webbink, R. F. 1985, in IAU Symp. 113, Dynamics of Star Clusters, ed. J. Goodman \& P. Hut (Cambridge: Cambridge Univ. Press), 541

Woosley, S. E., Wilson, J. R., Mathews, G. J., Hoffman, R. D., \& Meyer, B. S. 1994, ApJ, 433, 229

Yong, D., \& Grundahl, F. 2008, ApJ, 672, L29

Yong, D., Grundahl, F., D’Antona, F., Karakas, A. I., Lattanzio, J. C., \& Norris, J. E. 2009, ApJ, 695, L62

Yong, D., Karakas, A. I., Lambert, D. L., Chieffi, A., \& Limongi, M. 2008a, ApJ, 689, 1031

Yong, D., Lambert, D. L., Paulson, D. B., \& Carney, B. W. 2008b, ApJ, 673, 854

Zinn, R. 1973, ApJ, 182, 183

Zinn, R. 1980, ApJS, 42, 19 